BONN-TH-97-02

ITP Budapest Report No. 526

\title{
Coadjoint orbits of the Virasoro algebra and the global Liouville equation
}

\author{
J. Balog ${ }^{1, \dagger}$, L. Fehér ${ }^{2}$ and L. Palla ${ }^{3}$ \\ ${ }^{1}$ Laboratoire de Math. et Physique Théorique, CNRS UPRES-A 6083 \\ Université de Tours, Parc de Grandmont, F-37200 Tours, France \\ ${ }^{2}$ Physikalisches Institut der Universität Bonn, Nussallee 12, D-53115 Bonn, Germany \\ ${ }^{3}$ Institute for Theoretical Physics, Roland Eötvös University \\ H-1088 Budapest, Puskin u 5-7, Hungary
}

\begin{abstract}
The classification of the coadjoint orbits of the Virasoro algebra is reviewed and is then applied to analyze the so-called global Liouville equation. The review is selfcontained, elementary and is tailor-made for the application. It is well-known that the Liouville equation for a smooth, real field $\varphi$ under periodic boundary condition is a reduction of the $S L(2, \mathbf{R})$ WZNW model on the cylinder, where the WZNW field $g \in S L(2, \mathbf{R})$ is restricted to be Gauss decomposable. If one drops this restriction, the Hamiltonian reduction yields, for the field $Q=\kappa g_{22}$ where $\kappa \neq 0$ is a constant, what we call the global Liouville equation. Corresponding to the winding number of the $S L(2, \mathbf{R})$ WZNW model there is a topological invariant in the reduced theory, given by the number of zeros of $Q$ over a period. By the substitution $Q= \pm \exp (-\varphi / 2)$, the Liouville theory for a smooth $\varphi$ is recovered in the trivial topological sector. The nontrivial topological sectors can be viewed as singular sectors of the Liouville theory that contain blowing-up solutions in terms of $\varphi$. Since the global Liouville equation is conformally invariant, its solutions can be described by explicitly listing those solutions for which the stress-energy tensor belongs to a set of representatives of the Virasoro coadjoint orbits chosen by convention. This direct method permits to study the 'coadjoint orbit content' of the topological sectors as well as the behaviour of the energy in the sectors. The analysis confirms that the trivial topological sector contains special orbits with hyperbolic monodromy and shows that the energy is bounded from below in this sector only.
\end{abstract}

\footnotetext{
$\dagger$ On leave from the Research Institute for Particle and Nuclear Physics, Budapest, Hungary
} 


\section{Introduction}

The Liouville equation plays an important role in various areas of theoretical physics (for a review, see e.g. Ref. [1]). Our motivation for the present work was to explore the space of solutions of the evolution equation

$$
Q \partial_{+} \partial_{-} Q-\partial_{+} Q \partial_{-} Q=1, \quad x^{ \pm}=\frac{\tau \pm \sigma}{2}, \quad \partial_{ \pm}=\left(\partial_{\tau} \pm \partial_{\sigma}\right),
$$

for a smooth, real field ${ }^{a} Q$ under periodic boundary condition in $\sigma$. We call this equation the global Liouville equation, since it becomes the Liouville equation

$$
\partial_{+} \partial_{-} \varphi+2 e^{\varphi}=0
$$

upon the substitution $Q= \pm \exp (-\varphi / 2)$, which is valid only locally, if $Q$ has no zeros. Notice that the number of zeros of $Q$ over a period in $\sigma$ is a topological invariant for the global Liouville theory. It cannot be changed by a smooth deformation of $Q$, since (1.1) implies that $Q$ cannot have any double zero in $\sigma$. The number of zeros, $N$, is even as $Q$ is periodic. The Liouville equation (1.2) for a smooth $\varphi$ is recovered in the trivial, $N=0$ topological sector of (1.1), and the other sectors may be thought of as singular sectors of the Liouville theory, where the solutions blow up in terms of $\varphi$, but remain smooth in terms of $Q$. The smoothness of $Q$ is in fact equivalent to the smoothness of the conformally improved Liouville stress-energy tensor. One of the main questions about the model is how the energy behaves in the various topological sectors. It will turn out in the end that the energy is bounded from below in the trivial topological sector only.

The singular solutions of the Liouville equation were studied previously $[2,3]$ using methods different from those that will be used here. Equation (1.1) was apparently first introduced in the second article of Ref. [3]. We were led to the global Liouville equation by the earlier treatment [4] of the Liouville theory as a reduced Wess-Zumino-NovikovWitten (WZNW) model [5] on the cylinder for the group $S L(2, \mathbf{R})$. In that case, the Liouville equation arises if one further restricts the group valued field $g \in S L(2, \mathbf{R})$ to vary in the neighbourhood of the identity consisting of Gauss decomposable matrices. Dropping this restriction, one obtains precisely the global Liouville equation for $Q=\kappa g_{22}$, where $\kappa$ is the level parameter of the WZNW model. Hence, studying the solutions of (1.1) helps to explore the global structure of the phase space of the reduced WZNW model [6]. The topological sectors of the global Liouville theory correspond to the topological sectors of the WZNW model, which are labelled by the winding number of the field $g: S^{1} \times \mathbf{R} \rightarrow S L(2, \mathbf{R})$. The constraints of the reduction enforce that the winding number equals sign $\kappa$-times half the number of zeros of $g_{22}$.

\footnotetext{
${ }^{a}$ In this paper smooth means infinitely many times differentiable, i.e, $C^{\infty}$.
} 
Before explaining the content of the paper, we need to describe an algorithm for solving the global Liouville equation. The subsequent algorithm is well-known in the context of the Liouville equation, and is based on the observation that if one defines $L_{ \pm}:=$ $\partial_{ \pm}^{2} Q / Q$, then $\partial_{\mp} L_{ \pm}=0$ on account of (1.1). In terms of $\varphi, L_{ \pm}$are just the light cone components of the conformally improved Liouville stress-energy tensor. For a smooth $Q$ which is periodic in $\sigma, L_{ \pm}$are smooth, periodic functions depending respectively on $x^{ \pm}$ only. For convenience, the length of the period in $\sigma$ is set equal to $4 \pi$. As a first step for solving (1.1), one may therefore consider the pair of Schrödinger equations

$$
\left(\partial_{ \pm}^{2}-L_{ \pm}\right) \psi^{ \pm}=0
$$

for some given smooth $L_{ \pm}$with $L_{ \pm}\left(x^{ \pm}+2 \pi\right)=L_{ \pm}\left(x^{ \pm}\right)$. This is also known as Hill's equation [7]. If $\psi_{i}^{ \pm}\left(x^{ \pm}\right)$for $i=1,2$ are independent, real solutions normalized by the Wronskian condition

$$
\psi_{2}^{ \pm} \partial_{ \pm} \psi_{1}^{ \pm}-\psi_{1}^{ \pm} \partial_{ \pm} \psi_{2}^{ \pm}=1
$$

then

$$
Q\left(x^{+}, x^{-}\right):=\Psi_{+}\left(x^{+}\right) \Psi_{-}^{T}\left(x^{-}\right), \quad \text { where } \quad \Psi_{ \pm}:=\left(\begin{array}{ll}
\psi_{ \pm}^{1} & \psi_{ \pm}^{2}
\end{array}\right),
$$

solves (1.1). This solution is automatically smooth as well, but its periodicity in $\sigma$ constrains the admissible pairs $\left(L_{+}, L_{-}\right)$. Namely, since $L_{ \pm}$are periodic, the solutions of the Hill's equations are quasiperiodic with some monodromy, i.e.,

$$
\Psi_{ \pm}\left(x^{ \pm}+2 \pi\right)=\Psi_{ \pm}\left(x^{ \pm}\right) M_{\Psi_{ \pm}} \quad \text { for some } \quad M_{\Psi_{ \pm}} \in S L(2, \mathbf{R}) .
$$

The periodicity condition $Q\left(x^{+}, x^{-}\right)=Q\left(x^{+}+2 \pi, x^{-}-2 \pi\right)$ is clearly equivalent to

$$
M_{\Psi_{-}}=\left(M_{\Psi_{+}}\right)^{T} .
$$

Because the transformations

$$
\Psi_{+} \mapsto \Psi_{+} A, \quad \Psi_{-} \mapsto \Psi_{-}\left(A^{-1}\right)^{T} \quad A \in S L(2, \mathbf{R})
$$

do not change $Q$ and transform the monodromy matrices by conjugation, one may assume without loss of generality that $M_{\Psi_{+}}$belongs to a given set of representatives of the conjugacy classes of $S L(2, \mathbf{R})$ when writing the solutions of (1.1) in the form (1.5).

The global Liouville equation is conformally invariant, since if $Q$ is a solution then so is $Q^{\alpha_{+}, \alpha_{-}}$given by

$$
Q^{\alpha_{+}, \alpha_{-}}\left(x^{+}, x^{-}\right):=\frac{1}{\sqrt{\partial_{+} \alpha_{+}\left(x^{+}\right)}} \frac{1}{\sqrt{\partial_{-} \alpha_{-}\left(x^{-}\right)}} Q\left(\alpha_{+}\left(x^{+}\right), \alpha_{-}\left(x^{-}\right)\right)
$$

for any conformal transformation $x^{ \pm} \mapsto \alpha_{ \pm}\left(x^{ \pm}\right)$, where the smooth functions $\alpha_{ \pm}$satisfy

$$
\partial_{ \pm} \alpha_{ \pm}>0, \quad \alpha_{ \pm}\left(x^{ \pm}+2 \pi\right)=\alpha_{ \pm}\left(x^{ \pm}\right)+2 \pi .
$$


In order to describe all solutions of (1.1), it is enough to know the conformally nonequivalent ones. An important fact is that the action of the conformal group on $Q$ in (1.9) is induced from its action on the set of Hill's equations according to

$$
\begin{gathered}
L_{ \pm} \mapsto L_{ \pm}^{\alpha_{ \pm}}:=\left(\partial_{ \pm} \alpha_{ \pm}\right)^{2} L_{ \pm} \circ \alpha_{ \pm}-\frac{1}{2} \frac{\partial_{ \pm}^{3} \alpha_{ \pm}}{\partial_{ \pm} \alpha_{ \pm}}+\frac{3}{4} \frac{\left(\partial_{ \pm}^{2} \alpha_{ \pm}\right)^{2}}{\left(\partial_{ \pm} \alpha_{ \pm}\right)^{2}} \\
\Psi_{ \pm} \mapsto \Psi_{ \pm}^{\alpha_{ \pm}}:=\frac{1}{\sqrt{\partial_{ \pm} \alpha_{ \pm}}} \Psi_{ \pm} \circ \alpha_{ \pm} .
\end{gathered}
$$

For each chirality, the action in (1.11a) is just the coadjoint action of the central extension of the conformal group $\operatorname{Diff}_{0}\left(S^{1}\right)$. Its orbits in the space of 'Virasoro densities' $L_{ \pm}$ are known as the coadjoint orbits of the Virasoro algebra ${ }^{b}$. Equations (1.11a), (1.11b) together express the conformal covariance of Hill's equation (1.3).

It is now clear that the classification of the Hill's equations under the conformal group is essentially the same as the classification of the Virasoro coadjoint orbits, and this is the crucial step for describing the solutions of the global Liouville equation. Indeed, since all smooth, periodic solutions of (1.1) arise from the above algorithm, the conformally nonequivalent ones among these solutions of (1.1) can be written down explicitly once one has the list of conformally nonequivalent Virasoro densities, and the associated nonequivalent solutions of Hill's equation.

The classification of Virasoro coadjoint orbits is well-known $[8,9,10,11,12,13,14]$. However, we found that the expositions that are available in the literature are not explicit enough for what is needed in the study of the global Liouville equation. This is especially true regarding representatives for the nonequivalent solutions of Hill's equation. Therefore we have rederived the necessary classification keeping in mind its application to study (1.1). Our derivation is elementary, and we obtain complete proofs of all statements. Since our description of the classification might be useful in other applications too, we thought it worthwhile to publish it as a review, in which we included a comparison with the previous treatments of the problem.

The above-mentioned review takes up the bigger part of the paper, and then we come back to apply it to equation (1.1). More precisely, the paper is organized as follows. First, in Section 2, we summarize the WZNW description of the global Liouville system. The following section, which is the longest, is devoted to presenting the list of nonequivalent Hill's equations together with their explicit solutions. The reader is advised to consult Subsection 3.7 for a summary. Then, in Section 4, we describe the behaviour of the chiral energy functional (the zero mode of the Virasoro density) on the Virasoro coadjoint orbits. This was also studied in Ref. [11], and we complete the

${ }^{b}$ To be exact, the properly normalized Virasoro densities are $\mathcal{L}_{ \pm}=2 \kappa L_{ \pm}$. Geometrically, they belong to the hyperplane of centre $C=24 \pi \kappa$ in the dual of $\widehat{\operatorname{diff}}\left(S^{1}\right)$. See Section 2 . 
arguments of this reference on some minor points. In Section 5, we obtain an explicit list of solutions for the global Liouville equation, which in particular shows the exact relationship between the topological type (number of zeros) and the Virasoro orbit type of the solutions. Combining this with the results in Section 4, we establish the behaviour of the 'total energy' (defined as the reduced WZNW Hamiltonian) in the topological sectors of equation (1.1). Finally, we give our conclusions in Section 6, and there are 4 appendices containing technical details.

\section{Reduced WZNW treatment of the global Liouville equation}

We here recall $[4,6]$ the interpretation of the global Liouville system as a reduced WZNW model. This also serves to fix the conventions used in the subsequent sections.

Consider the WZNW model for the group $S L(2, \mathbf{R})$ in the Hamiltonian framework. The phase space of the model (the set of initial data at $\tau=0$ ) can be realized as the manifold

$$
\mathcal{M}_{\mathrm{WZ}}=\left\{\left(g, J_{+}\right) \mid g \in C^{\infty}\left(S^{1}, S L(2, \mathbf{R})\right), \quad J_{+} \in C^{\infty}\left(S^{1}, \operatorname{sl}(2, \mathbf{R})\right)\right\} .
$$

It comes equipped with the fundamental Poisson brackets

$$
\begin{aligned}
\{g(\sigma), g(\bar{\sigma})\}_{\mathrm{WZ}} & =0, \\
\left\{\operatorname{tr}\left(T_{a} J_{+}\right)(\sigma), g(\bar{\sigma})\right\}_{\mathrm{WZ}} & =-T_{a} g(\sigma) \delta_{4 \pi}, \quad \delta_{4 \pi}=\delta_{4 \pi}(\sigma-\bar{\sigma}), \\
\left\{\operatorname{tr}\left(T_{a} J_{+}\right)(\sigma), \operatorname{tr}\left(T_{b} J_{+}\right)(\bar{\sigma})\right\}_{\mathrm{WZ}} & =\operatorname{tr}\left(\left[T_{a}, T_{b}\right] J\right)(\sigma) \delta_{4 \pi}+2 \kappa \operatorname{tr}\left(T_{a} T_{b}\right) \delta_{4 \pi}^{\prime},
\end{aligned}
$$

where $\kappa \in \mathbf{R}$ is a nonzero constant, and $T_{a}$ is a basis of $\operatorname{sl}(2, \mathbf{R})$. The functions on $S^{1}$ are realized as periodic functions on $\mathbf{R}$ with period $4 \pi$ using the variable $\sigma$ that parametrizes $S^{1}$ by $z=\exp \left(\frac{i \sigma}{2}\right)$, and $\delta_{4 \pi}(\sigma)=\frac{1}{4 \pi} \sum_{n \in \mathbf{Z}} e^{i n \sigma / 2}$ is the $4 \pi$-periodic Dirac- $\delta$. Defining

$$
J_{-}=-g^{-1} J_{+} g+2 \kappa g^{-1} g^{\prime}
$$

the WZNW Hamiltonian

$$
\mathcal{H}_{\mathrm{WZ}}=\frac{1}{4 \kappa} \int_{0}^{4 \pi} d \sigma \operatorname{tr}\left(J_{+}^{2}+J_{-}^{2}\right)(\sigma)
$$

generates the evolution equation

$$
\kappa \partial_{+} g=J_{+} g, \quad \partial_{-} J_{+}=0 .
$$

The global Liouville system will result from reduction by the constraints ${ }^{c}$

$$
\operatorname{tr}\left(e_{12} J_{+}\right)=1, \quad \operatorname{tr}\left(e_{21} J_{-}\right)=-1,
$$

${ }^{c}$ We could equivalently set the currents in (2.6) equal to any constants $\mu_{ \pm}$with $\mu_{+} \mu_{-}<0$, while $\mu_{+} \mu_{-}>0$ would lead to a system containing the Liouville theory with wrong sign. 
where the $e_{i j}$ are the standard matrices having 1 for the $(i, j)$-entry and zero elsewhere. These constraints are first class and generate the gauge transformations

$$
g \rightarrow \exp \left(A e_{12}\right) g \exp \left(-B e_{21}\right), \quad J_{+} \rightarrow \exp \left(A e_{12}\right) J_{+} \exp \left(-A e_{12}\right)+2 \kappa A^{\prime} e_{12}
$$

for any $A, B \in C^{\infty}\left(S^{1}, \mathbf{R}\right)$. A globally well-defined gauge fixing is obtained by restricting the pair $\left(g, J_{+}\right)$in such a way that

$$
g=\left(\begin{array}{cc}
R & P_{+} \\
P_{-} & g_{22}
\end{array}\right), \quad J_{+}=\left(\begin{array}{cc}
0 & \ell_{+} \\
1 & 0
\end{array}\right), \quad \text { and } \quad J_{-}=-\left(\begin{array}{cc}
0 & 1 \\
\ell_{-} & 0
\end{array}\right)
$$

Due to $(2.3)$ these restrictions on $\left(g, J_{+}\right)$can be rewritten in the form

$$
R=\ell_{+} g_{22}-2 \kappa P_{+}^{\prime}, \quad P_{-}=P_{+}-2 \kappa g_{22}^{\prime}, \quad \ell_{-}=R^{2}-\ell_{+} P_{-}^{2}+2 \kappa\left(R^{\prime} P_{-}-R P_{-}^{\prime}\right)
$$

which express $R, P_{-}, \ell_{-}$in terms of $g_{22}, P_{+}, \ell_{+}$, and a remaining constraint

$$
1=\operatorname{det} g=g_{22}\left(\ell_{+} g_{22}-2 \kappa P_{+}^{\prime}\right)-P_{+}\left(P_{+}-2 \kappa g_{22}^{\prime}\right) \text {. }
$$

Thus we can think of the reduced WZNW phase space as the submanifold in the space of the triples $\left(g_{22}, P_{+}, \ell_{+}\right)$of smooth, periodic functions defined by $(2.10)$.

It is appropriate to comment here on the topological sectors of the reduced system. Denote by $N\left[g_{22}\right]$ the number of zeros of $g_{22}(\sigma)$ over a period $0 \leq \sigma<4 \pi$. Note from (2.10) that $g_{22}(\sigma)$ and $g_{22}^{\prime}(\sigma)$ cannot have simultaneous zeros:

$$
\left(g_{22}^{2}+\left(g_{22}^{\prime}\right)^{2}\right)(\sigma) \neq 0
$$

Therefore $g_{22}(\sigma)$ cannot have any double zero, thus $N\left[g_{22}\right]$ is even (possibly 0 ), and it defines a topological invariant of $g_{22}$. Consequently, the reduced WZNW phase space decomposes into disconnected components labelled by the values of $N\left[g_{22}\right]$. It is shown in Appendix A that the invariant $N\left[g_{22}\right]$ is inherited from the winding number $W[g]$ of $g: S^{1} \rightarrow S L(2, \mathbf{R})$, which is a topological invariant in the original WZNW model. The winding number is invariant with respect to the gauge group in $(2.7)$, since this group has trivial topology.

To describe the reduced WZNW dynamics, we note that the gauge fixing in (2.8) is preserved by the WZNW evolution equation (2.5), and therefore the reduced dynamics can be obtained by simply applying $(2.5)$ to the gauge fixed variables $\left(g, J_{+}\right)$in $(2.8)$. This yields the evolution equation

$$
\kappa \partial_{+} g_{22}=P_{+}, \quad \kappa \partial_{+} P_{+}=\ell_{+} g_{22}, \quad \partial_{-} \ell_{+}=0 .
$$

In terms of the variables $\left(g_{22}, P_{-}, \ell_{-}\right)$, the reduced dynamics is equivalently given by

$$
\kappa \partial_{-} g_{22}=P_{-}, \quad \kappa \partial_{-} P_{-}=\ell_{-} g_{22}, \quad \partial_{+} \ell_{-}=0 .
$$


Using (2.12-13) the constraint in (2.10) becomes

$$
\left(Q \partial_{+} \partial_{-} Q-\partial_{+} Q \partial_{-} Q\right)=1 \quad \text { where } \quad Q:=\kappa g_{22} \text {. }
$$

This is just our global Liouville equation in (1.1). Conversely, any smooth, periodic solution of (2.14) gives a solution of (2.12) by defining $P_{+}:=\partial_{+} Q$ and $\ell_{+}:=\kappa^{2} \partial_{+}^{2} Q / Q$. By this definition, (2.14) becomes just the constraint (2.10). The smoothness of $\ell_{+}$is also guaranteed since $\partial_{+}^{2} Q / Q=\partial_{+}^{2} \partial_{-} Q / \partial_{-} Q$, where $Q$ and $\partial_{-} Q$ cannot be simultaneously zero by (2.14). In conclusion, the global Liouville equation encodes the reduced WZNW dynamics.

We now wish to elaborate on the conformal symmetry of the reduced sytem. We first note that (2.12-13) imply $\partial_{ \pm}^{2} Q\left(x^{+}, x^{-}\right)=L_{ \pm}\left(x^{ \pm}\right) Q\left(x^{+}, x^{-}\right)$with

$$
L_{ \pm}\left(x^{ \pm}\right)=\frac{1}{\kappa^{2}} \ell_{ \pm}\left(\sigma= \pm 2 x^{ \pm}, \tau=0\right)
$$

Hence, comparison with (1.3), (1.5) allows us to identify the variables $L_{ \pm}$in (2.15) with the similarly named variables of Section 1 . As $\ell_{ \pm}$are functions on the reduced WZNW phase space that carries a natural induced Poisson bracket (the Dirac bracket), we can then compute the Poisson brackets of

$$
\mathcal{L}_{ \pm}\left(x^{ \pm}\right):=2 \kappa L_{ \pm}\left(x^{ \pm}\right)
$$

and find [4]

$$
\left\{\mathcal{L}_{ \pm}(x), \mathcal{L}_{ \pm}(y)\right\}=-\kappa \delta_{2 \pi}^{\prime \prime \prime}(x-y)+2 \mathcal{L}_{ \pm}(y) \delta_{2 \pi}^{\prime}(x-y)-\mathcal{L}_{ \pm}^{\prime}(y) \delta_{2 \pi}(x-y)
$$

where $\delta_{2 \pi}(x)=\frac{1}{2 \pi} \sum_{n \in \mathbf{Z}} e^{i n x}$ is the $2 \pi$-periodic Dirac- $\delta$. Defining the modes $\mathcal{L}_{ \pm}^{n}$ by

$$
\mathcal{L}_{ \pm}^{n}:=\int_{0}^{2 \pi} d x e^{i n x} \mathcal{L}_{ \pm}(x)+\kappa \pi \delta_{n, 0}, \quad \forall n \in \mathbf{Z}
$$

eq. (2.17) is equivalent to

$$
\left\{\mathcal{L}_{ \pm}^{n}, \mathcal{L}_{ \pm}^{m}\right\}=(-i)\left((n-m) \mathcal{L}_{ \pm}^{n+m}+24 \pi \kappa \frac{n\left(n^{2}-1\right)}{12} \delta_{n+m, 0}\right)
$$

These are two commuting copies of the Virasoro algebra with centre

$$
C=24 \pi \kappa
$$

The overall factor $(-i)$ on the r.h.s. of (2.19) reflects correctly the correspondence principle between Poisson brackets and commutators, and the second term has the standard 
form that vanishes for the Möbius subalgebras generated by $\mathcal{L}_{ \pm}^{-1}, \mathcal{L}_{ \pm}^{0}, \mathcal{L}_{ \pm}^{1}$ due to the shift in the definition of $\mathcal{L}_{ \pm}^{0}$ in (2.18).

The variation $\Delta_{\epsilon_{ \pm}}$of a dynamical variable under an infinitesimal conformal transformation $x^{ \pm} \mapsto x^{ \pm}+\epsilon_{ \pm}\left(x^{ \pm}\right)$is defined by its Poisson bracket with the respective charge $T_{\epsilon_{ \pm}}=\int_{0}^{2 \pi} d x \epsilon_{ \pm}(x) \mathcal{L}_{ \pm}(x)$. In particular, this gives

$$
\Delta_{\epsilon_{ \pm}} L_{ \pm}:=-\left\{T_{\epsilon_{ \pm}}, L_{ \pm}\right\}=\epsilon_{ \pm} L_{ \pm}^{\prime}+2 \epsilon_{ \pm}^{\prime} L_{ \pm}-\frac{1}{2} \epsilon_{ \pm}^{\prime \prime \prime},
$$

which we recognize to be the infinitesimal version of the transformation rule in (1.11a). It follows that as the variable $L_{ \pm}$maps out an orbit of the conformal group according to (1.11a), the variable $\mathcal{L}_{ \pm}$in (2.16) runs through a coadjoint orbit of the Virasoro algebra at centre $C=24 \pi \kappa$. The advantage of using the variables $L_{ \pm}$is that their transformation rule is independent of $C$, and this allows one to describe the Virasoro coadjoint orbits at any $C \neq 0$ at one stroke. We shall use this device in the subsequent sections, but for proper interpretation the relationship (2.16) must be remembered.

Let us further observe that the WZNW Hamiltonian (2.4) survives the reduction because its restriction to the constrained manifold (2.6) is gauge invariant. This gauge invariant function defines the natural Hamiltonian for the reduced WZNW model, that is for the global Liouville system. The Hamiltonian is of course constant along any solution of the reduced dynamics. Denoting its value by $\mathcal{H}_{\mathrm{wz}}[Q]$ we easily verify that

$$
\mathcal{H}_{\mathrm{WZ}}[Q]=\frac{1}{2}\left(\mathcal{L}_{+}^{0}+\mathcal{L}_{-}^{0}\right)-\kappa \pi,
$$

where $\mathcal{L}_{ \pm}^{0}$ are defined in (2.18). On account of (2.16), it is convenient to represent the Virasoro zero modes in the form

$$
\mathcal{L}_{ \pm}^{0}=4 \pi \kappa\left(E\left[L_{ \pm}\right]+\frac{1}{4}\right) \quad \text { where } \quad E\left[L_{ \pm}\right]=\frac{1}{2 \pi} \int_{0}^{2 \pi} d x L_{ \pm}(x) .
$$

Later we will study the question of boundedness from below for the "chiral energy functionals' $E\left[L_{ \pm}\right]$on the Virasoro coadjoint orbits, and apply the result to understand the behaviour of the 'total energy' $\mathcal{H}_{\mathrm{WZ}}[Q]$ in the global Liouville system.

\section{The normal forms of Hill's equation}

In this section, we obtain the classification of the Virasoro coadjoint orbits by analysing the Hill's equation associated with the Virasoro densities. We first define a rough classification by attaching to each Virasoro orbit the conjugacy class of the monodromy matrix of the corresponding Hill's equation. We then find the conformally nonequivalent Virasoro densities, and the solutions of Hill's equation with any given monodromy. The main ideas and the results of this classification are known, but our presentation is different, since we list explicit representatives for the nonequivalent objects. 


\subsection{Statement of the problem and recall of some known results}

We are interested in the classification of the Hill's equations

$$
\psi^{\prime \prime}=L \psi,
$$

where $L$ is a smooth, real, periodic function on the line $\mathbf{R}$ with period $2 \pi$, with respect to the group of transformations

$$
\begin{gathered}
L \mapsto L^{\alpha}:=\alpha^{\prime 2} L \circ \alpha+S(\alpha), \quad S(\alpha):=-\frac{1}{2} \frac{\alpha^{\prime \prime \prime}}{\alpha^{\prime}}+\frac{3}{4} \frac{\alpha^{\prime \prime 2}}{{\alpha^{\prime 2}}^{2}}, \\
\psi \mapsto \psi^{\alpha}:=\frac{1}{\sqrt{\alpha^{\prime}}} \psi \circ \alpha,
\end{gathered}
$$

where $\alpha$ is a smooth, real function on $\mathbf{R}$ with the property

$$
\alpha^{\prime}>0, \quad \alpha(x+2 \pi)=\alpha(x)+2 \pi .
$$

The mappings $\alpha: \mathbf{R} \rightarrow \mathbf{R}$ with this property form a subgroup of the group $\operatorname{Diff}_{0}(\mathbf{R})$ of

orientation preserving diffeomorphisms of the line. This group is denoted by $\widetilde{\operatorname{Diff}}_{0}\left(S^{1}\right)$ since it is a covering group of the group of orientation preserving diffeomorphisms of the circle $\operatorname{Diff}_{0}\left(S^{1}\right)$. The homomorphism

$$
\chi: \widetilde{\operatorname{Diff}}_{0}\left(S^{1}\right) \rightarrow \operatorname{Diff}_{0}\left(S^{1}\right)
$$

is naturally induced from the map $\mathbf{R} \rightarrow S^{1}$ given by $x \mapsto e^{i x}$. The kernel of $\chi$ is the group of translations on $\mathbf{R}$ by integer multiples of $2 \pi$. Formula (3.2) defines an action of $\widetilde{\operatorname{Diff}}_{0}\left(S^{1}\right)$ on the set of Hill's equations, and we wish to find a list of 'normal forms' of $L$ and those of the corresponding solutions $\psi$ that are nonequivalent under this group.

The action of $\widetilde{\operatorname{Diff}}_{0}\left(S^{1}\right)$ in (3.2a) on the space of $L$ 's factors through the homomorphism $\chi$ to an action of $\operatorname{Diff}_{0}\left(S^{1}\right)$. By identifying the space of $L$ 's with a hyperplane at centre $C \neq 0$ in the dual of the Virasoro Lie algebra, this action can be recognized as the coadjoint action of the central extension of $\operatorname{Diff}_{0}\left(S^{1}\right)$, which at the infinitesimal level is the coadjoint action of the Virasoro Lie algebra (see e.g. [11]). Thus our problem to classify the orbits of the action in (3.2) is essentially equivalent to the classification of the Virasoro coadjoint orbits $[8,9,10,11,12,13,14]$. We shall present the solution in an approach convenient for application to equation (1.1).

We wish to stress here that in this paper we often call the orbits of the group action in (3.2a) Virasoro coadjoint orbits, even though it is $\mathcal{L}=\frac{C}{12 \pi} L$ which is the properly normalized Virasoro density (see eqs. (2.16)-(2.21)). In this way, we effectively describe the coadjoint orbits at an arbitrary $C \neq 0$ in $C$-independent terms, and this slight abuse of notation should not lead to any misunderstanding. 
For purposes of comparison between our approach, which is close to Refs. [8,10,12], and the one described by Kirillov [9] and Witten [11], we now recall some known results. First note that the Lie algebra of the little group $G[L] \subset \operatorname{Diff}_{0}\left(S^{1}\right)$ of $L$ is spanned by the $2 \pi$-periodic vector fields $\xi(x) \frac{\partial}{\partial x}$ (infinitesimal conformal transformations) that solve

$$
\xi^{\prime \prime \prime}-4 L \xi^{\prime}-2 L^{\prime} \xi=0,
$$

and if $\psi_{1}, \psi_{2}$ are the independent solutions of (3.1), then the solutions of (3.5) are the products

$$
\psi_{1}^{2}, \quad \psi_{2}^{2}, \quad \psi_{1} \psi_{2},
$$

which admit either 1 or 3 periodic linear combinations [7,9]. The coadjoint orbit $\mathcal{O}_{L}$ through $L$ can be represented as

$$
\mathcal{O}_{L}=\operatorname{Diff}_{0}\left(S^{1}\right) / G[L]=\widetilde{\operatorname{Diff}_{0}}\left(S^{1}\right) / \tilde{G}[L],
$$

where

$$
\tilde{G}[L]:=\chi^{-1}(G[L]) \subset \widetilde{\operatorname{Diff}_{0}}\left(S^{1}\right)
$$

is the lift of $G[L] \subset \operatorname{Diff}_{0}\left(S^{1}\right)$. In the terminology of Ref. [11], the nonconjugate little groups $G[L] \subset \operatorname{Diff}_{0}\left(S^{1}\right)$ that define the possible orbits $\mathcal{O}_{L}$ are $[9,11]$

$$
S^{1}, \quad P S L^{(n)}(2, \mathbf{R}), \quad T_{n, \Delta}, \quad \tilde{T}_{n, \pm} \quad(n \in \mathbf{N}, \quad \Delta>0) .
$$

Here $S^{1}$ is the group of rigid rotations of $S^{1} ; P S L^{(n)}(2, \mathbf{R})$ is a subgroup of $\operatorname{Diff}_{0}\left(S^{1}\right)$ isomorphic to the $n$-fold cover of the projective group $P S L(2, \mathbf{R})=S L(2, \mathbf{R}) / Z_{2} ; T_{n, \Delta}$ and $\tilde{T}_{n, \pm}$ are special one dimensional subgroups whose Lie algebras are given by vector fields on $S^{1}$ having $2 n$ simple and, respectively, $n$ double zeros. The groups $T_{n, \Delta}$ and $\tilde{T}_{n, \pm}$ are not connected, since they also contain certain discrete subgroups isomorphic to the group $\mathbf{Z}_{n}$ of rigid rotations by multiples of the angle $2 \pi / n$. These little groups classify the coadjoint orbits up to diffeomorphisms, but conjugate little groups may belong to nonequivalent points in the space of $L$ 's. This actually happens only for the orbit $\operatorname{Diff}_{0}\left(S^{1}\right) / S^{1}$, which carries a one parameter family of nonequivalent symplectic structures at fixed value of the central parameter.

The subgroup $\tilde{G}[L]$ in (3.8) still acts on the solutions of Hill's equation at the standard point $L$ of $\mathcal{O}_{L}$, and this has to be taken into account when listing the conformally nonequivalent solutions. We shall present a complete list of conformally nonequivalent solutions of Hill's equation under the additional assumptions that the solutions are normalized by a Wronskian condition and that they have nonconjugate monodromy matrices. The monodromy matrix will be fixed to vary in a set of representatives of the conjugacy classes of $S L(2, \mathbf{R})$ chosen in the next subsection. 


\subsection{Rough classification by conjugacy classes of the monodromy matrix}

For given $L$, let $\psi_{i}(i=1,2)$ be independent, real solutions of Hill's equation normalized by the Wronskian condition

$$
\psi_{2} \psi_{1}^{\prime}-\psi_{2}^{\prime} \psi_{1}=1
$$

The solution space of (3.1) is two-dimensional and the solution vector ${ }^{d} \Psi$ given by

$$
\Psi:=\left(\begin{array}{ll}
\psi_{1} & \psi_{2}
\end{array}\right)
$$

is determined up to

$$
\Psi \mapsto \Psi A, \quad \forall A \in S L(2, \mathbf{R}) .
$$

Since $L$ is $2 \pi$-periodic, the translation operator

$$
\Psi \mapsto \tilde{\Psi}, \quad \tilde{\Psi}(x):=\Psi(x+2 \pi)
$$

acts on the solutions, and it obviously preserves the Wronskian condition (3.10). Thus we can associate the monodromy matrix $M_{\Psi} \in S L(2, \mathbf{R})$ to $\Psi$ by

$$
\tilde{\Psi}=\Psi M_{\Psi} .
$$

The monodromy matrix enjoys the property

$$
M_{\Psi A}=A^{-1} M_{\Psi} A, \quad \forall A \in S L(2, \mathbf{R}) .
$$

This means that the conjugacy class of the monodromy matrix depends only on the potential $L$ defining the Hill's equation (3.1), and is independent of the freedom in (3.12). Moreover, if $\Psi$ is a solution vector of the Hill's equation at $L$, then

$$
\Psi^{\alpha}:=\left(\begin{array}{ll}
\psi_{1}^{\alpha} & \psi_{2}^{\alpha}
\end{array}\right)
$$

is a solution vector at $L^{\alpha}$ (it satisfies the Wronskian condition) with

$$
M_{\Psi^{\alpha}}=M_{\Psi} .
$$

In conclusion, the conjugacy class of the monodromy matrix is a conformally invariant function of $L$.

Considering the action of the little group $\tilde{G}[L]$ in (3.8), (3.17) implies that

$$
\Psi^{\alpha^{-1}}=\Psi \gamma(\alpha) \quad \forall \alpha \in \tilde{G}[L],
$$

\footnotetext{
${ }^{d}$ Solution basis would be a more correct term, but we often operate on $\Psi$ like on a 2-vector.
} 
where $\gamma(\alpha)$ belongs to the little group $G\left[M_{\Psi}\right] \subset S L(2, \mathbf{R})$ of the monodromy matrix. For any fixed $\Psi$, the map

$$
\gamma: \tilde{G}[L] \rightarrow G\left[M_{\Psi}\right]
$$

is actually a homomorphism (this is why we used the inverse in the definition (3.18a)). The normalized solution vectors of Hill's equation at the standard point $L \in \mathcal{O}_{L}$, with fixed monodromy matrix $M_{\Psi}$ are in one-to-one correspondence with the elements $g$ of $G\left[M_{\Psi}\right]$, by $g \leftrightarrow \Psi g$. The orbits of the little group $\tilde{G}[L]$ in the space of these solution vectors can therefore be parametrized by the points of the coset space

$$
\gamma(\tilde{G}[L]) \backslash G\left[M_{\Psi}\right]
$$

We shall see that this space consists of a single point in most cases, but at most 2 points.

Now let $\mathcal{M}$ be the set of conjugacy classes of $S L(2, \mathbf{R})$ and

$$
p: S L(2, \mathbf{R}) \rightarrow \mathcal{M}
$$

be the canonical projection. Denote by $\mathcal{V}$ the set of the Virasoro coadjoint orbits $\mathcal{O}_{L}$. We have a well-defined map from $\mathcal{V}$ to $\mathcal{M}$ given by the formula

$$
\mathcal{O}_{L} \mapsto p\left(M_{\Psi}\right)
$$

This map is many-to-one, and yields a rough classification of the Virasoro coadjoint orbits. To describe it more concretely, we below present a list of representatives for the conjugacy classes of $S L(2, \mathbf{R})$. There exist 4 types of conjugacy classes: elliptic, hyperbolic, parabolic and one-point classes. The elliptic and hyperbolic classes are generic since they contain the elements $g \in S L(2, \mathbf{R})$ for which $|\operatorname{tr}(g)|<2$ and $|\operatorname{tr}(g)|>2$, respectively. Our terminology is somewhat nonstandard concerning the remaining special classes, as we call parabolic only those that contain more than one element.

Our representatives are as follows. First, a hyperbolic conjugacy class is represented by a matrix $B_{\eta}(b)$ of the form

$$
B_{\eta}(b):=\eta\left(\begin{array}{cc}
e^{2 \pi b} & 0 \\
0 & e^{-2 \pi b}
\end{array}\right), \quad \eta= \pm 1, \quad b>0 .
$$

The corresponding little group $G\left[B_{\eta}(b)\right] \subset S L(2, \mathbf{R})$ contains the matrices

$$
\left(\begin{array}{cc}
\beta & 0 \\
0 & 1 / \beta
\end{array}\right), \quad \beta \neq 0 .
$$

Second, an elliptic class is represented by a matrix $C(\omega)$ of the form

$$
C(\omega):=\left(\begin{array}{cc}
\cos \omega & -\sin \omega \\
\sin \omega & \cos \omega
\end{array}\right), \quad 0<\omega<\pi, \quad \pi<\omega<2 \pi .
$$


The little group $G[C(\omega)]$ contains the matrices

$$
\left(\begin{array}{cc}
\cos \vartheta & -\sin \vartheta \\
\sin \vartheta & \cos \vartheta
\end{array}\right), \quad 0 \leq \vartheta<2 \pi .
$$

Third, one has the 2 one-point classes given by

$$
E_{\eta}:=\eta\left(\begin{array}{ll}
1 & 0 \\
0 & 1
\end{array}\right), \quad \eta= \pm 1,
$$

whose little group is the full group $G\left[E_{\eta}\right]=S L(2, \mathbf{R})$. Finally, one has the 4 parabolic classes represented by the matrices $P_{\eta}^{q}$,

$$
P_{\eta}^{q}:=\eta\left(\begin{array}{ll}
1 & 0 \\
q & 1
\end{array}\right), \quad \eta= \pm 1, \quad q= \pm 1 .
$$

The little group $G\left[P_{\eta}^{q}\right]$ is the maximal nilpotent subgroup consisting of the matrices

$$
\left(\begin{array}{cc} 
\pm 1 & 0 \\
\gamma & \pm 1
\end{array}\right), \quad \forall \gamma \in \mathbf{R}
$$

In addition to the monodromy parameter furnished by the map (3.21), the classification of the Virasoro coadjoint orbits requires an additional discrete parameter [10,12]. The orbits associated with monodromy conjugacy classes of type $B, C, E, P$ will be denoted by the letters $\mathcal{B}, \mathcal{C}, \mathcal{E}, \mathcal{P}$, respectively, plus parameters needed for their complete specification. This will be related to the notation in Refs. [8,9,11].

\subsection{Virasoro coadjoint orbits with elliptic monodromy}

If $L$ is such that the monodromy of Hill's equation is elliptic, choose a solution vector $\Psi=\left(\begin{array}{ll}\psi_{1} & \psi_{2}\end{array}\right)$ so that the monodromy matrix is of the form $C(\omega)$ in $(3.23 \mathrm{a})$. Then

$$
\mathcal{R}:=\psi_{1}^{2}+\psi_{2}^{2}>0
$$

is a periodic function, which is a well-defined functional of $L$, since it is unchanged under $\Psi \mapsto \Psi A_{0}, A_{0} \in G[C(\omega)]$. It is straightforward to verify the equality

$$
L=\frac{\psi_{1}^{\prime \prime} \psi_{1}+\psi_{2}^{\prime \prime} \psi_{2}}{\psi_{1}^{2}+\psi_{2}^{2}}=\frac{1}{2} \frac{\mathcal{R}^{\prime \prime}}{\mathcal{R}}-\frac{1}{4} \frac{\mathcal{R}^{\prime 2}}{\mathcal{R}^{2}}-\frac{1}{\mathcal{R}^{2}}
$$

It is also clear that

$$
\nu:=\frac{1}{\pi} \int_{0}^{2 \pi} \frac{d y}{\mathcal{R}(y)}, \quad \nu>0,
$$

is invariant under the conformal group (3.2), and that

$$
\alpha(x):=\frac{2}{\nu} \int_{0}^{x} \frac{d y}{\mathcal{R}(y)}
$$


satisfies (3.3). With this $\alpha,(3.27)$ can be rewritten as the equality

$$
L=\alpha^{\prime 2}\left(-\frac{\nu^{2}}{4}\right)+S(\alpha)
$$

By (3.2), this means that $L$ lies on the Virasoro coadjoint orbit through

$$
L_{\nu}:=-\frac{\nu^{2}}{4}
$$

i.e., $L=L_{\nu}^{\alpha}$. Hence the 'standard Virasoro densities' $L_{\nu}$ provide a complete, nonredundant set of representatives of the Virasoro coadjoint orbits with elliptic monodromy.

The solution vector $\Psi_{\nu}=\left(\begin{array}{ll}\psi_{1, \nu} & \psi_{2, \nu}\end{array}\right)$ of Hill's equation at $L_{\nu}$, with standard monodromy, is given by

$$
\psi_{1, \nu}(x)=\sqrt{\frac{2}{\nu}} \sin \frac{\nu x}{2}, \quad \psi_{2, \nu}(x)=\sqrt{\frac{2}{\nu}} \cos \frac{\nu x}{2},
$$

up to the freedom contained in the little group $G[C(\omega)]$. Indeed, the corresponding monodromy matrix is $C(\omega)$ with

$$
\omega=\nu \pi-2 \pi d \quad \text { for some } \quad d \in \mathbf{Z}_{+}:=\{0\} \cup \mathbf{N} .
$$

This establishes the relationship between $\nu$ and the monodromy parameter $\omega$, and one sees that the latter determines the former up to a nonnegtive integer $d$. The integer just mentioned may be regarded as the discrete parameter that labels the orbits with elliptic monodromy in addition to $\omega$. Note that $\nu \notin \mathbf{N}$ since we have elliptic monodromy (3.23a).

The little group $G\left[L_{\nu}\right] \subset \operatorname{Diff}_{0}\left(S^{1}\right)$ is in fact $[9,11]$ the group of rigid rotations of $S^{1}$, which lifts to the translation group of $\mathbf{R}$, i.e., $\tilde{G}\left[L_{\nu}\right]=\mathbf{R}$ (see also Appendix B). One can easily evaluate the action of this group on the solution vector $\Psi_{\nu}$ in $(3.32)$, which shows that the orbit of $\tilde{G}\left[L_{\nu}\right]$ contains all solution vectors at $L_{\nu}$ with the same monodromy matrix, i.e., the coset space in (3.19) consists of a single point in this case. We conclude that the solution in (3.32) is a representative for all solutions of Hill's equation with monodromy $C(\omega)$ and fixed value of the invariant $\nu$ in (3.28). In particular, since $L=L_{\nu}^{\alpha}$, we can write

$$
\psi_{1}=\psi_{1, \nu}^{\alpha}=\sqrt{\frac{2}{\nu \alpha^{\prime}}} \sin \frac{\nu}{2} \alpha, \quad \psi_{2}=\psi_{2, \nu}^{\alpha}=\sqrt{\frac{2}{\nu \alpha^{\prime}}} \cos \frac{\nu}{2} \alpha
$$

up to the little group of conformal transformations.

To summarize, the Virasoro coadjoint orbits with elliptic monodromy are

$$
\mathcal{C}(\nu):=\mathcal{O}_{L_{\nu}}=\operatorname{Diff}_{0}\left(S^{1}\right) / S^{1}=\widetilde{\operatorname{Diff}_{0}}\left(S^{1}\right) / \mathbf{R}, \quad \nu>0, \quad \nu \notin \mathbf{N}
$$


and, for any $L \in \mathcal{C}(\nu)$, the Wronskian-normalized solutions of Hill's equation with monodromy matrix $C(\omega)$ are conformally equivalent to the solution in (3.32). The orbits in (3.35) associated with different values of $\nu$ are diffeomorphic, but of course this does not mean equivalence from the phase space (symplectic form, energy etc) point of view.

\subsection{Virasoro coadjoint orbits with $E_{\eta}$-type monodromy}

The analogues of equations (3.26-32) are valid in this case as well. We find that any $L$ of monodromy type $E_{\eta}$ in (3.24) lies on a Virasoro orbit through $L_{\nu}=-\frac{\nu^{2}}{4}$ for some positive $\nu$. The monodromy matrix of the normalized solution vector $(3.32)$ at $L_{\nu}$ becomes the matrix $E_{\eta}$ if $\nu$ is an integer. Therefore we have

$$
\nu=n \in \mathbf{N} \quad \text { and } \quad \eta=(-1)^{n}
$$

A difference from the elliptic case is that $\mathcal{R}$ in (3.26) is not unique in the present case, since now the solution vector $\Psi$ can be transformed according to

$$
\Psi \mapsto \Psi A, \quad \forall A \in G\left[E_{\eta}\right]=S L(2, \mathbf{R})
$$

and $\mathcal{R}$ is not invariant under such a transformation in general.

The Lie algebra of the little group $G\left[L_{n}\right] \subset \operatorname{Diff}_{0}\left(S^{1}\right)$ is spanned by the vector fields

$$
\frac{\partial}{\partial x}, \quad \cos n x \frac{\partial}{\partial x}, \quad \sin n x \frac{\partial}{\partial x}
$$

which define nonconjugate embeddings of $\operatorname{sl}(2, \mathbf{R})$ into the Lie algebra of $\operatorname{Diff}_{0}\left(S^{1}\right)$ for different values of $n$. Globally $[9,11]$ (see also Appendix B), $G\left[L_{n}\right]$ is the $n$-fold covering group of $P S L(2, \mathbf{R})$, denoted by $P S L^{(n)}(2, \mathbf{R})$. Then equations (3.8), (3.18) yield a homomorphism

$$
\gamma: \chi^{-1}\left(P S L^{(n)}(2, \mathbf{R})\right) \rightarrow S L(2, \mathbf{R})
$$

which is surjective, since it is just the standard homomorphism of the universal covering group of $S L(2, \mathbf{R})$ onto $S L(2, \mathbf{R})$. A particular consequence is that, despite the noninvariance of $\mathcal{R}$ under (3.37),

$$
n=\frac{1}{\pi} \int_{0}^{2 \pi} \frac{d y}{\psi_{1}^{2}+\psi_{2}^{2}}
$$

is independent from the choice of the solution vector $\Psi$, as it should be. This integral is obviously invariant under conformal transformations, and this implies its invariance under (3.37), since the latter transformations arise from conformal transformations by the surjective homomorphism (3.39). Another consequence is that the coset space (3.19) consists of a single point for $M_{\Psi}=E_{\eta}$. 
In conclusion, the Virasoro coadjoint orbits with monodromy matrix $E_{\eta}$ are

$$
\mathcal{E}_{n}:=\mathcal{O}_{L_{n}}=\operatorname{Diff}_{0}\left(S^{1}\right) / P S L^{(n)}(2, \mathbf{R})=\widetilde{\operatorname{Diff}_{0}}\left(S^{1}\right) / \chi^{-1}\left(P S L^{(n)}(2, \mathbf{R})\right)
$$

where $n \in \mathbf{N}$ and $\eta=(-1)^{n}$. The Wronskian-normalized solutions of Hill's equation (3.1) at $L \in \mathcal{E}_{n}$ are conformally equivalent to the solution $\Psi_{n}$ at $L_{n}$ given by

$$
\psi_{1, n}(x)=\sqrt{\frac{2}{n}} \sin \frac{n x}{2}, \quad \psi_{2, n}(x)=\sqrt{\frac{2}{n}} \cos \frac{n x}{2} .
$$

\subsection{Virasoro coadjoint orbits with hyperbolic monodromy}

If $L$ is such that the monodromy of Hill's equation is hyperbolic, consider a solution vector $\Psi=\left(\begin{array}{ll}\psi_{1} & \psi_{2}\end{array}\right)$ whose monodromy matrix takes the standard form $B_{\eta}(b)$ in (3.22a). Then associate a nonnegative integer $n$ to $L$ by means of ${ }^{e}$

$$
L \mapsto n(L):=\text { number of zeros of } \psi_{2}(x) \text { for } 0 \leq x<2 \pi .
$$

Since $\Psi$ is unique up to

$$
\Psi \mapsto \Psi A, \quad A \in G\left[B_{\eta}(b)\right],
$$

the map $L \mapsto n(L)$ is well-defined. Furthermore, it follows from the transformation rule (3.2) that $n(L)$ is a conformally invariant function on the space of $L$ 's with hyperbolic monodromy. The discrete invariant $n \in \mathbf{Z}_{+}$determines the invariant $\eta$ appearing in the specification of the monodromy matrix,

$$
\eta=(-1)^{n} .
$$

Below, we show that the monodromy invariant $b>0$ and $n$ together provide a complete classification of the Virasoro coadjoint orbits with hyperbolic monodromy, which may therefore be labelled as

$$
\mathcal{B}_{n}(b), \quad b>0, \quad n \in \mathbf{Z}_{+} .
$$

We proceed by proving that any $L$ with invariants $b, n$ can be brought to a standard form by a conformal transformation. We first deal with the simplest case.

3.5.1. The case $\mathcal{B}_{0}(b)$. Since $\psi_{2}$ has no zeros, the function $u$ defined by

$$
u:=\frac{\psi_{1}}{\psi_{2}}
$$

is smooth in this case. This function satisfies

$$
u^{\prime}=\frac{1}{\psi_{2}^{2}}>0 \quad \text { and } \quad u(x+2 \pi)=e^{4 \pi b} u(x),
$$

\footnotetext{
$e$ Because of the Wronskian condition, one could equivalently use $\psi_{1}$ in the definition.
} 
and hence it is monotonically increasing with limits

$$
\lim _{x \rightarrow-\infty} u(x)=0, \quad \lim _{x \rightarrow+\infty} u(x)=+\infty
$$

These properties of $u$ permit to define an element $\alpha \in \widetilde{\operatorname{Diff}}_{0}\left(S^{1}\right)$ by

$$
\alpha:=\frac{1}{2 b} \ln u
$$

It is then straightforward to verify the identity

$$
L=\frac{\psi_{2}^{\prime \prime}}{\psi_{2}}=b^{2}\left(\alpha^{\prime}\right)^{2}+S(\alpha),
$$

proving that $L$ lies on the orbit of the 'standard point'

$$
L_{2 i b}:=b^{2}
$$

The little group $G\left[L_{2 i b}\right]$ is the group of rigid rotations of $S^{1}$. Therefore we have

$$
\mathcal{B}_{0}(b)=\mathcal{O}_{L_{2 i b}}=\operatorname{Diff}_{0}\left(S^{1}\right) / S^{1}=\widetilde{\operatorname{Diff}}_{0}\left(S^{1}\right) / \mathbf{R}
$$

where we used that

$$
\tilde{G}\left[L_{2 i b}\right]=\mathbf{R}
$$

is the translation group of $\mathbf{R}$. We further claim that the conformally nonequivalent solutions of Hill's equation at $L_{2 i b}$ are given by $\Psi^{ \pm}=\left(\begin{array}{ll}\psi_{1}^{ \pm} & \psi_{2}^{ \pm}\end{array}\right)$, where

$$
\psi_{1}^{ \pm}(x)= \pm \frac{1}{\sqrt{2 b}} e^{b x}, \quad \psi_{2}^{ \pm}(x)= \pm \frac{1}{\sqrt{2 b}} e^{-b x} .
$$

Indeed, the image of the little group (3.54) under the homomorphism (3.18) is the connected component of the identity in $G\left[B_{\eta}(b)\right]$, and thus the coset space (3.19) now consists of 2 points. As a consequence, the solutions of Hill's equation with monodromy matrix $B_{+}(b)$ at any $L \in \mathcal{B}_{0}(b)$ are conform transforms of $\Psi^{ \pm}$.

3.5.2. The case $\mathcal{B}_{n}(b)$ for $n \in \mathbf{N}$. We start by noting that if $\Psi=\left(\begin{array}{ll}\psi_{1} & \psi_{2}\end{array}\right)$ is a pair of smooth functions subject to the Wronskian condition (3.10), then

$$
L:=\frac{\psi_{1}^{\prime \prime}}{\psi_{1}}=\frac{\psi_{2}^{\prime \prime}}{\psi_{2}}
$$

is also smooth. If in addition

$$
\Psi(x+2 \pi)=\Psi(x) B_{\eta}(b)
$$


then $L$ is periodic as well. In other words, $\Psi$ is a solution vector of Hill's equation at $L$.

To show that Virasoro coadjoint orbits with $n(L) \neq 0$ exist, it is enough to present examples. For arbitrarily chosen $b>0$ and $n \in \mathbf{N}$, let us define

$$
\begin{aligned}
& \psi_{1}(x):=\frac{e^{b x}}{\sqrt{F(x)}} \sqrt{\frac{n}{2}}\left(\frac{2 b}{n^{2}} \cos \frac{n x}{2}+\frac{2}{n} \sin \frac{n x}{2}\right) \\
& \psi_{2}(x):=\frac{e^{-b x}}{\sqrt{F(x)}} \sqrt{\frac{2}{n}} \cos \frac{n x}{2}
\end{aligned}
$$

where

$$
F(x)=F_{n, b}(x):=\cos ^{2} \frac{n x}{2}+\left(\sin \frac{n x}{2}+\frac{2 b}{n} \cos \frac{n x}{2}\right)^{2} .
$$

Since the function $F$ has no zeros, $\Psi$ is smooth. It is clear that $\psi_{2}$ has $n$ zeros, and (3.10), (3.57) are satisfied. Thus $\Psi$ is a solution vector of Hill's equation at $L_{n, b}:=L$ given by (3.56), which explicitly reads as

$$
L_{n, b}=b^{2}+\frac{n^{2}+4 b^{2}}{2 F}-\frac{3}{4} \frac{n^{2}}{F^{2}} .
$$

This Virasoro density and the solution $\Psi$ in (3.58) are deformations of the $\mathcal{E}_{n}$-type representatives $L_{n}=-\frac{n^{2}}{4}$ and $\Psi_{n}$ in (3.42) that are recovered in the limit $b=0$.

We now claim that any Virasoro density with hyperbolic monodromy and discrete invariant $n \in \mathbf{N}$ lies on the orbit $\mathcal{B}_{n}(b)$ of the 'standard point' $L_{n, b}$ in (3.59). The idea of the proof is as follows. For an arbitrary $\bar{L}$ with the same monodromy and discrete invariants as $L_{n, b}$, let $\bar{\Psi}=\left(\begin{array}{ll}\bar{\psi}_{1} & \bar{\psi}_{2}\end{array}\right)$ be a normalized solution vector at $\bar{L}$ with monodromy matrix $B_{\eta}(b)$. Using that both $\psi_{2}$ in (3.58) and $\bar{\psi}_{2}$ have $n$ zeros, it is not hard to see that $\bar{\psi}_{2}$ can be transformed into $\psi_{2}$ by a conformal transformation, i.e.,

$$
\exists \alpha \in \widetilde{\operatorname{Diff}}_{0}\left(S^{1}\right) \quad \text { such that } \frac{1}{\sqrt{\alpha^{\prime}}} \bar{\psi}_{2}(\alpha(x))=\psi_{2}(x) .
$$

A construction of the required $\alpha$ is contained in Appendix C. Once (3.60) is proven, $L=\bar{L}^{\alpha}$ follows from Hill's equation. Since $\bar{\psi}_{2}$ uniquely determines $\bar{\psi}_{1}$ by the Wronskian and monodromy conditions, this argument also proves that the solution $\bar{\Psi}$ is conformally equivalent to the solution $\Psi$ in (3.58).

The little group $G\left[L_{n, b}\right] \subset \operatorname{Diff}_{0}\left(S^{1}\right)$ is spanned by the vector field $\xi(x) \frac{\partial}{\partial x}$, where

$$
\xi(x)=\psi_{1}(x) \psi_{2}(x)=\cos \frac{n x}{2}\left(\frac{2 b}{n^{2}} \cos \frac{n x}{2}+\frac{2}{n} \sin \frac{n x}{2}\right) / F(x)
$$

is now the only periodic one among the expressions in (3.6). This vector field has $2 n$ simple zeros over the period $0 \leq x<2 \pi$, and therefore we can identify $G\left[L_{n, b}\right]$ in terms of the list in $(3.9)$ as

$$
G\left[L_{n, b}\right] \simeq T_{n, \Delta}
$$


Using the explicit expression in (3.58), the relation between the monodromy invariant $b$ and the invariant $[9,11] \Delta$ can be obtained from the definition [11] of $\Delta$ as

$$
\Delta=4 \pi b .
$$

Observe from (3.59) that $L_{n, b}$ is periodic with period $\frac{2 \pi}{n}$. Thus $G\left[L_{n, b}\right] \simeq T_{n, 4 \pi b}$ contains the cyclic group $\mathbf{Z}_{n}$ of rigid rotations on $S^{1}$ by multiples of the angle $\frac{2 \pi}{n}$. The structure of the little group is further clarified in Appendix C. We find that up to isomorphism

$$
G\left[L_{n, b}\right]=\mathbf{R}_{+} \times \mathbf{Z}_{n} \quad \text { and } \quad \tilde{G}\left[L_{n, b}\right]=\mathbf{R}_{+} \times \mathbf{T}_{\frac{2 \pi}{n}}
$$

where $\mathbf{R}_{+}$is the multiplicative group of positive real numbers and $\mathbf{T}_{\frac{2 \pi}{n}}$ is the group of translations on $\mathbf{R}$ by multiples of $\frac{2 \pi}{n}$. This is consistent with the fact that $\gamma$ in (3.18) is a surjective homomorphism onto $G\left[B_{\eta}(b)\right] \simeq \mathbf{R}_{+} \times \mathbf{Z}_{2}$. The structure of $T_{n, \Delta} \simeq G\left[L_{n, b}\right]$ has not been fully clarified in Refs. [9,11], and our result (3.63) is in conflict with a claim of Ref. [14] on this point.

To summarize, we have established the identification

$$
\mathcal{B}_{n}(b)=\mathcal{O}_{L_{n, b}}=\operatorname{Diff}_{0}\left(S^{1}\right) / G\left[L_{n, b}\right]=\operatorname{Diff}_{0}\left(S^{1}\right) / T_{n, 4 \pi b}
$$

and proved that up to conformal transformations $\Psi$ in (3.58) represents the solutions of Hill's equation with monodromy $B_{\eta}(b), \eta=(-1)^{n}$ along this Virasoro coadjoint orbit.

\subsection{Virasoro coadjoint orbits with parabolic monodromy}

The discussion is similar to that in the previous subsection. For any $L$ for which the monodromy matrix of Hill's equation belongs to the conjugacy class of $P_{\eta}^{q}$ in $(3.25 \mathrm{a})$, consider a solution vector $\Psi=\left(\begin{array}{ll}\psi_{1} & \psi_{2}\end{array}\right)$ whose monodromy matrix equals $P_{\eta}^{q}$. It follows from $(3.25 \mathrm{~b})$ that $\psi_{2}$ is now unique up to sign. Hence the discrete invariant $n(L)$ defined by (3.43) can again be attached to the Virasoro coadjoint orbit through $L$, and $\eta=(-1)^{n}$ holds. Below, we show that the invariants $n \in \mathbf{Z}_{+}$and $q= \pm 1$ together provide a complete classification of the Virasoro orbits in this case. The orbit corresponding to a fixed value of $n \in \mathbf{Z}_{+}$and $q \in\{ \pm 1\}$ will be denoted by $\mathcal{P}_{n}^{q}$. Sometimes we also write $\mathcal{P}_{n}^{ \pm}$to refer to $\mathcal{P}_{n}^{q}$ for $q= \pm 1$, respectively. The list of orbits turns out to be

$$
\mathcal{P}_{0}^{+}, \quad \mathcal{P}_{n}^{q} \quad n \in \mathbf{N}, \quad q \in\{ \pm 1\} .
$$

If $n=0$, then only the value $q=+1$ occurs. We justify our claim by bringing any $L$ with invariants $n, q$ to a standard form by a conformal transformation.

3.6.1. The case $\mathcal{P}_{0}^{+}$. Choosing any $\Psi$ with monodromy $P_{1}^{q}$, the smooth function $u=\frac{\psi_{1}}{\psi_{2}}$ now satisfies

$$
u^{\prime}=\frac{1}{\psi_{2}^{2}}>0 \quad \text { and } \quad u(x+2 \pi)=u(x)+q
$$


where $q=+1$ since $u$ is monotonically increasing. This explains the remark after equation (3.65), and also implies that $\alpha:=2 \pi u$ is an element of $\widetilde{\operatorname{Diff}}_{0}\left(S^{1}\right)$. As a consequence of an analogous general property of $\frac{\psi_{1}}{\psi_{2}}$, one then obtains

$$
L=\frac{\psi_{2}^{\prime \prime}}{\psi_{2}}=-\frac{1}{2} \frac{u^{\prime \prime \prime}}{u^{\prime}}+\frac{3}{4} \frac{u^{\prime \prime 2}}{u^{\prime 2}}=S(u)=S(\alpha),
$$

which means by (3.2) that $L$ is on the coadjoint orbit of

$$
L_{0}(x):=0
$$

It is clear from (3.6) that the Lie algebra of the the little group $G\left[L_{0}\right] \subset \operatorname{Diff}_{0}\left(S^{1}\right)$ is spanned by the vector field $\frac{\partial}{\partial x}$, and $G\left[L_{0}\right]=S^{1}$ in fact. At $L_{0}$, two conformally nonequivalent solution vectors of Hill's equation are given by $\Psi_{0}^{ \pm}=\left(\begin{array}{ll}\psi_{1,0}^{ \pm} & \psi_{2,0}^{ \pm}\end{array}\right)$:

$$
\begin{aligned}
& \psi_{1,0}^{ \pm}(x):= \pm \frac{x}{\sqrt{2 \pi}} \\
& \psi_{2,0}^{ \pm}(x):= \pm \sqrt{2 \pi} .
\end{aligned}
$$

One readily confirms that the image of the homomorphism $\gamma: \tilde{G}\left[L_{0}\right] \rightarrow G\left[P_{1}^{1}\right]$ defined by (3.18) is the connected component of $G\left[P_{1}^{1}\right]$. Thus $\Psi_{0}^{ \pm}$represent the conformally nonequivalent solutions of Hill's equation with monodromy $P_{1}^{1}$ along the coadjoint orbit

$$
\mathcal{P}_{0}^{+}=\mathcal{O}_{L_{0}}=\operatorname{Diff}_{0}\left(S^{1}\right) / S^{1}=\widetilde{\operatorname{Diff}}_{0}\left(S^{1}\right) / \mathbf{R} .
$$

3.6.2. The case $\mathcal{P}_{n}^{q}$ for $n \in \mathbf{N}$. For any $q \in\{ \pm 1\}, n \in \mathbf{N}$ and $\eta=(-1)^{n}$, define $\Psi_{n, q}=\left(\begin{array}{ll}\psi_{1, n, q} & \psi_{2, n, q}\end{array}\right)$ by

$$
\begin{aligned}
& \psi_{1, n, q}(x):=\frac{1}{\sqrt{H(x)}}\left(\frac{q x}{2 \pi} \sin \frac{n x}{2}-\frac{2}{n} \cos \frac{n x}{2}\right) \\
& \psi_{2, n, q}(x):=\frac{1}{\sqrt{H(x)}} \sin \frac{n x}{2}
\end{aligned}
$$

where

$$
H(x)=H_{n, q}(x):=1+\frac{q}{2 \pi} \sin ^{2} \frac{n x}{2} .
$$

This is a solution vector of Hill's equation with monodromy $P_{\eta}^{q}$ and $n$ zeros of $\psi_{2}$ at

$$
L_{n, q}=\frac{n^{2}}{2 H}-\frac{3 n^{2}\left(1+\frac{q}{2 \pi}\right)}{4 H^{2}} .
$$

The proof in Appendix $\mathrm{C}$ shows that any Virasoro density $L$ of the same monodromy type and discrete invariant $n(L)$ lies on the coadjoint orbit of $L_{n, q}$, i.e., we have

$$
\mathcal{P}_{n}^{q}=\mathcal{O}_{L_{n, q}}=\operatorname{Diff}_{0}\left(S^{1}\right) / G\left[L_{n, q}\right]
$$


The Lie algebra of $G\left[L_{n, q}\right]$ is spanned by the vector field

$$
\xi(x) \frac{\partial}{\partial x}, \quad \xi(x)=\psi_{2, n, q}^{2}(x) .
$$

Since $\xi(x)$ has $n$ double zeros, we can identify the little group in terms of the list (3.9) as

$$
G\left[L_{n, q}\right] \simeq \tilde{T}_{n, \operatorname{sign}(-q)},
$$

where the flip of sign is merely our convention for defining $\tilde{T}_{n, \pm}$. Similarly to $G\left[L_{n, b}\right]$ in (3.63), $G\left[L_{n, q}\right]$ has the structure $\mathbf{R}_{+} \times \mathbf{Z}_{n}$ and (3.18) gives a surjective homomorphism. The solution $\Psi_{n, q}$ in (3.71) is conformally equivalent to any solution vector of Hill's equation that has the same monodromy matrix $P_{\eta}^{q}, \eta=(-1)^{n}$ and discrete invariant $n$.

\subsection{Summary of the Virasoro coadjoint orbits}

In this section, we described the coadjoint orbits of the Virasoro algebra by exhibiting representatives for all of them, and presented an explicit list for the conformally nonequivalent solutions of Hill's equation. We also provided the link between our description of the coadjoint orbits and the one based on the inspection of the possible little groups $[9,11]$ of the Virasoro densities. In our notation, the set of orbits is given by

$$
\mathcal{C}(\nu), \quad \mathcal{B}_{0}(b), \quad \mathcal{P}_{0}^{+} ; \quad \mathcal{E}_{n} ; \quad \mathcal{B}_{n}(b) ; \quad \mathcal{P}_{n}^{ \pm} \quad(n \in \mathbf{N}, \quad b>0),
$$

where we have arranged the orbits into four families according to the type of the little group in (3.9). Ref. [13] contains related (but incomplete) results on the connection between the monodromy invariant (3.21) and the type of the little group $G[L] \subset \operatorname{Diff}_{0}\left(S^{1}\right)$. Observe that the first two families in (3.76) consist of the orbits $\mathcal{O}_{L}$ possessing a constant representative $L=\Lambda$ for some $\Lambda \in \mathbf{R}$.

As a mnemonic, we find it useful to associate the set of Virasoro orbits in (3.76) with the points of a 'comb-like' figure, which we colloquially refer to as the 'Vircomb' (see Figure 1). To explain the shape of the Vircomb, recall that the $\mathcal{B}_{n}(b)$ representatives in (3.58) become the $\mathcal{E}_{n}$ ones in $(3.42)$ as $b \rightarrow 0$. As for the $\mathcal{P}_{n}^{q}$ orbits, they are also deformations of the $\mathcal{E}_{n}$ orbits in a sense [11]. To illustrate this, consider for example the one parameter curve of Virasoro densities $L_{n, q ; a}$ and corresponding solution vectors $\widehat{\Psi}_{n, q ; a}$ given as follows:

$$
L_{n, q ; a}:=\frac{n^{2} a^{2}}{2 H_{n, q ; a}}-\frac{3 n^{2} a^{2}\left(a^{2}+\frac{q}{2 \pi}\right)}{4 H_{n, q ; a}^{2}} \quad \text { with } \quad H_{n, q ; a}:=a^{2}+\frac{q}{2 \pi} \sin ^{2} \frac{n x}{2}
$$

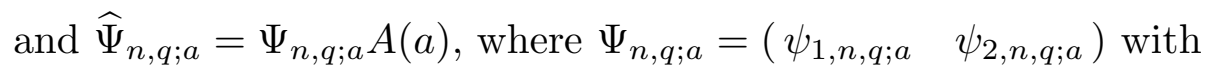

$$
\begin{aligned}
& \psi_{1, n, q ; a}(x):=\frac{1}{\sqrt{H_{n, q ; a}(x)}}\left(\frac{q x}{2 \pi} \sin \frac{n x}{2}-\frac{2 a^{2}}{n} \cos \frac{n x}{2}\right) \\
& \psi_{2, n, q ; a}(x):=\frac{1}{\sqrt{H_{n, q ; a}(x)}} \sin \frac{n x}{2}
\end{aligned}
$$


and

$$
A(a):=\left(\begin{array}{cc}
0 & -\frac{1}{a} \sqrt{\frac{n}{2}} \\
a \sqrt{\frac{2}{n}} & 0
\end{array}\right) .
$$

The parameter $a$ runs either as $a>0$ or as $a>1 / \sqrt{2 \pi}$ depending on whether $q=+1$ or $q=-1$, respectively. One can check that the monodromy matrix of $\widehat{\Psi}_{n, q ; a}$ belongs to the class of $P_{\eta}^{q}$ for any finite $a$ and the discrete invariant takes the value $n$, which ensure that the curve $L_{n, q ; a}$ lies on the orbit $\mathcal{P}_{n}^{q}$. The point we wish to make is that $L_{n, q ; a}$ has $L_{n}=-\frac{n^{2}}{4}$ as its $a \rightarrow \infty$ limit, and the solution vector $\widehat{\Psi}_{n, q ; a}$ becomes the $\mathcal{E}_{n}$ representative $\Psi_{n}(3.42)$ in this limit. This curve will be used in an argument later, too.

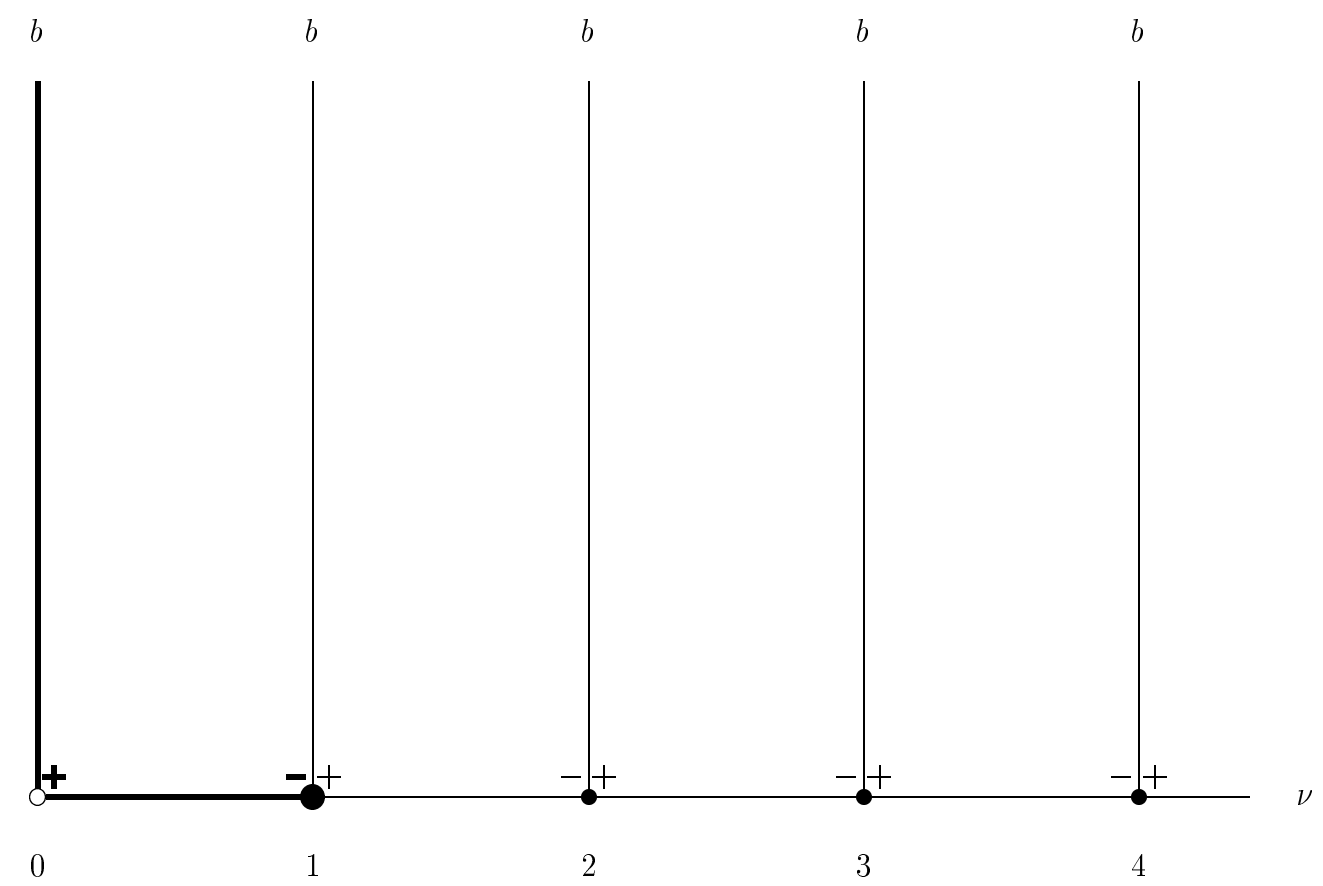

Figure 1: The coadjoint orbits of the Virasoro algebra.. The points of the figure are in oneto-one correspondence with the Virasoro coadjoint orbits at any fixed $C \neq 0$. The vertical lines (parametrized by $b$ ) correspond to the hyperbolic orbits with $n=0,1,2 \ldots$ The noninteger points on the horizontal axis correspond to the elliptic orbits, while the integer points (denoted by full circles) represent the $\mathcal{E}$-type orbits. The + and - signs around the integer points stand for the corresponding parabolic orbits. (Note that the open circle at $n=0$ is an empty point with no corresponding orbit.) The thickness of the vertical line at $n=0$ and the horizontal line between $n=0$ and $n=1$ as well as the $+\operatorname{sign}$ at $n=0$ and the - sign at $n=1$ and the larger radius of the circle at $n=1$ indicate that these are the only orbits for which the chiral energy functional $E[L]$ is bounded from below.

The approach whereby we derived the list in (3.76) is essentially an elementary version and elaboration of the approach followed in the previous papers $[8,10,12]$, where the classification of the Virasoro orbits was also presented as a refinement of the rough classification defined by the monodromy invariant (3.21). We now give a more detailed 
comparison with the results of Lazutkin and Pankratova [8]. These authors first define the following decomposition of the set $X$ of the Hill's equations in (3.1):

$$
X=X^{\text {stable }} \cup X^{\text {unstable }} \cup X^{\text {semistable }},
$$

where $X^{\text {stable }}$ consists of the equations that possess only bounded solutions, $X^{\text {unstable }}$ the equations whose nontrivial solutions are all unbounded, and $X^{\text {semistable }}$ is the rest. In our notation, $X^{\text {stable }}$ corresponds to elliptic and $E$ type monodromies, $X^{\text {unstable }}$ corresponds to hyperbolic monodromy, and $X^{\text {semistable }}$ corresponds to parabolic monodromy. They next consider a second decomposition $X=X^{\text {nonosc }} \cup X^{\text {osc }}$, where $X^{\text {osc }}$ contains the equations whose real solutions have infinitely many zeros both for positive and negative $x$. In terms of (3.76), we can identify these sets as

$$
X^{\text {nonosc }}=\left\{\mathcal{P}_{0}^{+}, \mathcal{B}_{0}(b)\right\} \quad \text { and } \quad X^{\text {osc }}=\left\{\mathcal{C}(\nu), \mathcal{E}_{n}, \mathcal{B}_{n}(b), \mathcal{P}_{n}^{ \pm}\right\}
$$

In effect, the elements of $X^{\text {osc }}$ are labelled in Ref. [8] by an invariant $\theta(L)$ together with the monodromy class in $P S L(2, \mathbf{R})$. The definition of the Lazutkin-Pankratova invariant $\theta(L)$, which unifies our invariants $\nu(L)$ and $n(L)$ that we defined in a case by case manner, is particularly elegant. For this one uses the translation number $\Theta(\alpha)$ associated to any $\alpha \in \widetilde{\operatorname{Diff}}_{0}\left(S^{1}\right)$ by $\Theta(\alpha):=\lim _{m \rightarrow \infty} \frac{1}{2 \pi m} \alpha^{m}(x)$, where $\alpha^{m}=\alpha \circ \alpha^{m-1}$. According to Poincaré, the limit exists and is independent of $x$. Then $\theta(L):=\Theta\left(\alpha_{L}\right)$, where $\alpha_{L}$ is defined as follows. Let $\psi^{x}$ be a nontrivial real solution of Hill's equation that vanishes at $x$, and set $\alpha_{L}(x)$ to be the zero of $\psi^{x}$ which is next to $x$ in the positive direction (such a zero exists since $\psi^{x}$ is oscillating). It is not difficult to verify that

$$
\theta(L)=\frac{1}{\nu} \quad \text { if } \quad L \in \mathcal{C}(\nu) \quad \text { and } \quad \theta(L)=\frac{1}{n} \quad \text { if } \quad L \in \mathcal{E}_{n}, \mathcal{B}_{n}(b), \mathcal{P}_{n}^{ \pm} \text {for } n \in \mathbf{N}
$$

This completes the correspondence between our classification and that in Ref. [8]. We note that Lazutkin and Pankratova did not give explicit representatives for the solutions of (3.1) in the nontrivial cases $\mathcal{P}_{n}^{ \pm}, \mathcal{B}_{n}(b)$, and it is not clear [10] whether their suggested representative Virasoro densities are correct in these cases or not.

\section{The energy functional on the coadjoint orbits}

In the previous section we described the coadjoint orbits of the Virasoro algebra at central charge $C \neq 0$. We now recall that Witten [11] also investigated the behaviour of the zero mode functional $\mathcal{L}^{0}$ on the orbits. An interesting question, motivated for example by analogy with the representation theory of the Virasoro algebra, is to find the list of orbits on which $\mathcal{L}^{0}$ is bounded from below. As was already mentioned, the zero mode functional is given by

$$
\mathcal{L}^{0}=\frac{C}{6}\left(E[L]+\frac{1}{4}\right) \quad \text { where } \quad E[L]=\frac{1}{2 \pi} \int_{0}^{2 \pi} d x L(x)
$$


is the so called chiral energy functional. On the orbit $\mathcal{O}_{L}$ of $L$ fixed, it is further convenient to define

$$
E_{L}[\alpha]:=E\left[L^{\alpha}\right], \quad \alpha \in \widetilde{\operatorname{Diff}_{0}}\left(S^{1}\right) .
$$

This functional is unbounded from above on every orbit (as is easily seen from (4.5)), and hence $\mathcal{L}^{0}$ is bounded from below if and only if $E_{L}[\alpha]$ is bounded from below and $C>0$. In fact, the orbits on which the chiral energy functional is bounded from below are:

$$
\mathcal{B}_{0}(b), \quad \mathcal{P}_{0}^{+}, \quad \mathcal{C}(\nu) \text { for } 0<\nu<1, \quad \mathcal{E}_{1}, \quad \mathcal{P}_{1}^{-},
$$

see Figure 1. Except for $\mathcal{P}_{1}^{-}$, these orbits contain a constant representative,

$$
L=\Lambda \quad \text { for } \quad \Lambda \geq-\frac{1}{4},
$$

and the chiral energy has a global minimum on the orbit taken precisely at the constant representative. This representative may be called a 'classical highest weight state' of the Virasoro algebra if $C>0$, since then $\mathcal{L}^{0}$ is also bounded from below by its global minimum taken at the classical highest weight state. The global minimum of $\mathcal{L}^{0}$ is positive except for the 'vacuum orbit' $\mathcal{E}_{1}$, where it takes the value zero. On the orbit $\mathcal{P}_{1}^{-}$ with $C>0$, the zero mode functional $\mathcal{L}^{0}$ has again the greatest lower bound given by zero, but this value is not taken on the orbit. These results were all described in Ref. [11], but in some cases (the case of $\mathcal{P}_{1}^{-}$and the question of global minimum at $L=\Lambda$ in (4.4)) no attempt was made to prove them. Our aim below is to present a complete, elementary proof. We shall proceed in a case by case manner.

\subsection{The case of the vacuum orbit $\mathcal{E}_{1}$}

As a preparation, let us spell out (4.2) as

$$
E_{L}[\alpha]=\frac{1}{2 \pi} \int_{0}^{2 \pi} d y\left\{\alpha^{\prime 2}(y) L(\alpha(y))+\frac{1}{4} \frac{\alpha^{\prime \prime 2}(y)}{\alpha^{\prime 2}(y)}\right\},
$$

where we used

$$
S(\alpha)=-\frac{1}{2} \frac{\alpha^{\prime \prime \prime}}{\alpha^{\prime}}+\frac{3}{4} \frac{\alpha^{\prime \prime 2}}{\alpha^{\prime 2}}=-\frac{1}{2}\left(\frac{\alpha^{\prime \prime}}{\alpha^{\prime}}\right)^{\prime}+\frac{1}{4} \frac{\alpha^{\prime \prime 2}}{\alpha^{2}} .
$$

Introducing the new integration variable

$$
y=\alpha^{-1}(x), \quad d y=\frac{d x}{q(x)} \quad \text { with } \quad q=\frac{1}{\left(\alpha^{-1}\right)^{\prime}}=\alpha^{\prime} \circ \alpha^{-1}
$$

eq. (4.5) becomes

$$
E_{L}[\alpha]=\frac{1}{2 \pi} \int_{0}^{2 \pi} d x\left\{q(x) L(x)+\frac{1}{4} \frac{q^{\prime 2}(x)}{q(x)}\right\}
$$


From its definition above, $q$ is a smooth, $2 \pi$-periodic function satisfying

$$
q>0, \quad \frac{1}{2 \pi} \int_{0}^{2 \pi} \frac{d x}{q(x)}=1 .
$$

Conversely, any $2 \pi$-periodic function $q$ subject to (4.9) determines a conformal transformation $\alpha^{-1}$, which is unique up to translations. Therefore we may regard $q$ with these properties as the independent variable in our extremum problem. (When doing so, we will denote the chiral energy (4.8) as $E_{L}[q]$.) Note also that if $\alpha \in \widetilde{\operatorname{Diff}}_{0}\left(S^{1}\right)$ is the lift of a Möbius transformation on $S^{1}$,

$$
e^{i x} \mapsto \frac{a e^{i x}+\bar{b}}{b e^{i x}+\bar{a}},
$$

where

$$
\left(\begin{array}{cc}
a & \bar{b} \\
b & \bar{a}
\end{array}\right) \in S U(1,1), \quad \text { i.e. } \quad|a|^{2}-|b|^{2}=1,
$$

then the corresponding function $q=1 /\left(\alpha^{-1}\right)^{\prime}$ has the form

$$
q(x)=\sqrt{1+\mu^{2}}-\mu \cos \left(x+x_{0}\right) \quad \text { with } \quad \mu=2|a b| \geq 0, \quad x_{0}=\arg b-\arg a .
$$

With this preparation in hand, we are ready to prove that on the orbit

$$
\mathcal{E}_{1}=\mathcal{O}_{L_{1}} \quad L_{1}=-\frac{1}{4},
$$

the chiral energy functional

$$
E_{L_{1}}[q]=\frac{1}{8 \pi} \int_{0}^{2 \pi} d x\left\{\frac{q^{\prime 2}}{q}-q\right\}
$$

has the global mininum $-1 / 4$, and the minimum is taken at those functions $q(x)$ that are of the form (4.12). Since the Möbius transformations leave $L_{1}$ invariant, the second statement means that the global minimum of the chiral energy on $\mathcal{E}_{1}$ is precisely at the 'classical vacuum state' $L_{1}$. We start by integrating the inequality

$$
\frac{1}{2 \pi}\left(\frac{\left|q^{\prime}\right|}{\sqrt{q}}-\sqrt{m+M-q-\frac{m M}{q}}\right)^{2} \geq 0
$$

which, using the definition (4.14) and the constraint (4.9), gives

$$
4 E_{L_{1}}[q] \geq-m-M+m M+\frac{1}{\pi} \int_{0}^{2 \pi} d x \frac{\left|q^{\prime}\right|}{q} \sqrt{\left(\frac{M-m}{2}\right)^{2}-\left(q-\frac{M+m}{2}\right)^{2}} .
$$

Here $m$ and $M$ are the absolute minimum and maximum of the function $q(x)$, respectively. 
If there were no absolute value sign in the integrand in (4.16), we could change the integration variable from $x$ to $q$. We can still do this piecewise, between two consecutive local extrema of the function $q(x)$, since within such an interval the sign of $q^{\prime}$ remains constant. We can now express the right hand side of (4.16) with the help of the primitive function of the integrand,

$$
\mathcal{F}(q)=\int_{m}^{q} \frac{d z}{z} \sqrt{\left(\frac{M-m}{2}\right)^{2}-\left(z-\frac{M+m}{2}\right)^{2}} .
$$

Let us look at this in detail for the case illustrated by Figure 2. (The general case can be treated similarly.) In this case we have

$$
\begin{aligned}
& 4 E_{L_{1}}[q] \geq-m-M+m M+\frac{2}{\pi}\left[\mathcal{F}\left(q_{1}\right)+\mathcal{F}\left(q_{3}\right)+\mathcal{F}\left(q_{5}\right)-\mathcal{F}\left(q_{2}\right)-\mathcal{F}\left(q_{4}\right)-\mathcal{F}\left(q_{6}\right)\right] \\
& =-m-M+m M+\frac{2}{\pi}[\mathcal{F}(M)-\mathcal{F}(m)]+\frac{2}{\pi}\left[\mathcal{F}\left(q_{3}\right)-\mathcal{F}\left(q_{4}\right)\right]+\frac{2}{\pi}\left[\mathcal{F}\left(q_{1}\right)-\mathcal{F}\left(q_{6}\right)\right] \\
& \geq-m-M+m M+\frac{2}{\pi}[\mathcal{F}(M)-\mathcal{F}(m)] \\
& =m M-2 \sqrt{m M}=(\sqrt{m M}-1)^{2}-1 \geq-1
\end{aligned}
$$

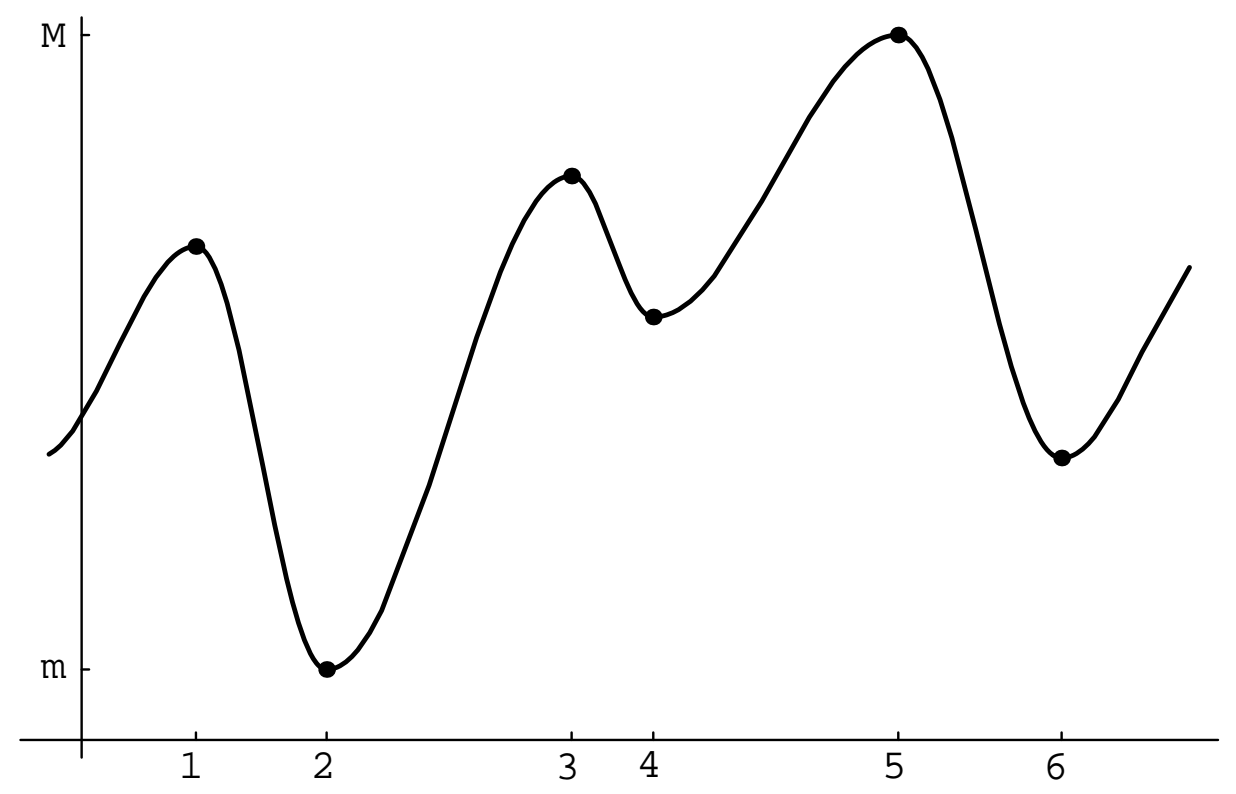

Figure 2: An example of the function $q(x)$

This shows that $-1 / 4$ is a lower bound for the functional $E_{L_{1}}[q]$. It is also clear from the derivation that this lower bound is saturated only if the following three conditions are satisfied by the function $q(x)$ :

1) It satisfies the differential equation

$$
\frac{q^{\prime 2}}{q}=m+M-q-\frac{m M}{q} .
$$


2) In addition to the points where $q(x)$ takes its absolute minimum $m$ and maximum $M$ it has no other local extrema.

3)

$$
m M=1 .
$$

The most general solution of the above conditions is given by (4.12). It is easy to check that (4.12) satisfies the requirements 1)-3) above. To see that it gives the most general solution it is useful to rewrite (4.19) in terms of $\beta:=\alpha^{-1}, q=1 / \beta^{\prime}$. One obtains that the derivative of (4.19) using (4.20) becomes the condition

$$
0=q^{\prime}\left(L_{1}^{\beta}-L_{1}\right) .
$$

It is well-known (and is shown in Appendix B) that the solutions of

$$
L_{1}^{\beta}=L_{1}
$$

are the Möbius transformations, yielding (4.12) as remarked earlier. Note that (4.22) may also be derived directly from (4.5) as the Euler-Lagrange equation of the functional $E_{L_{1}}[\alpha]$. This already implies that there is a unique local extremum of the chiral energy at $L_{1}$, but the above reasoning also proves that this really is a global minimum, which is not obvious [11].

\subsection{Arbitrary orbit with a constant representative}

Consider the orbit $\mathcal{O}_{\Lambda}$ of a constant $L$,

$$
L=\Lambda, \quad \Lambda \in \mathbf{R} .
$$

We wish to show that the behaviour of the chiral energy $E_{\Lambda}[\alpha]$ depends on whether $\Lambda \geq-\frac{1}{4}$ or $\Lambda<-\frac{1}{4}$. (Recall that $\mathcal{O}_{\Lambda}$ is of type $\mathcal{B}_{0}(b), \mathcal{P}_{0}^{+}, \mathcal{C}(\nu)$ or $\mathcal{E}_{n}$ depending on the particular value of the constant $\Lambda$.) For any $\Lambda$, one has the identity:

$$
E_{\Lambda}[\alpha]=\frac{\Lambda+\frac{1}{4}}{2 \pi} \int_{0}^{2 \pi} d x \alpha^{\prime 2}(x)+E_{-\frac{1}{4}}[\alpha]=\Lambda+\frac{\Lambda+\frac{1}{4}}{2 \pi} \int_{0}^{2 \pi} d x\left(\alpha^{\prime}-1\right)^{2}+\left(E_{-\frac{1}{4}}[\alpha]+\frac{1}{4}\right) .
$$

If one now takes

$$
\Lambda>-\frac{1}{4}
$$

then (4.24) and the result of the previous subsection imply that

$$
E_{\Lambda}[\alpha] \geq \Lambda
$$


and equality holds if and only if $\alpha^{\prime}=1$, which holds precisely for the elements of the little group of $L=\Lambda$. This proves that in the case (4.25) the chiral energy has a unique, global minimum on the orbit $\mathcal{O}_{\Lambda}$ taken at $L=\Lambda$, like for $\Lambda=-\frac{1}{4}$.

In contrast, if

$$
\Lambda<-\frac{1}{4}
$$

then the chiral energy is not bounded from below on the orbit $\mathcal{O}_{\Lambda}$. To see this, consider the one parameter subgroup of the Möbius group given by

$$
\left(\begin{array}{cc}
\cosh t & i \sinh t \\
-i \sinh t & \cosh t
\end{array}\right) \in S U(1,1), \quad \forall t \in \mathbf{R}
$$

for which one has

$$
q_{t}(x)=\cosh 2 t-\sinh 2 t \sin x .
$$

One then finds that

$$
E_{\Lambda}\left[q_{t}\right]=-\frac{1}{4}+\left(\Lambda+\frac{1}{4}\right) \cosh 2 t .
$$

Since $t$ is arbitrary, the chiral energy is indeed not bounded from below in the case (4.27).

\subsection{The orbits $\mathcal{P}_{n}^{-}$for $n \geq 2$ and $\mathcal{P}_{n}^{+}, \mathcal{B}_{n}(b)$ for any $n \in \mathbf{N}$}

We wish to show that the chiral energy is not bounded from below on these orbits. First, consider the orbit $\mathcal{P}_{n}^{-}$, whose representative $L_{n,-}$ is defined by (3.71-72) with $q=-1$. One can verify the inequality

$$
L_{n,-}(x)<-\frac{n^{2}}{8}
$$

Therefore

$$
L_{n,-}(x)<-\frac{1}{2} \quad \text { if } \quad n \geq 2
$$

which implies by comparison with a constant $L=\Lambda$ in (4.27) that the chiral energy is not bounded from below in this case.

Second, using the curve $L_{n,+; a}$ of Virasoro densities on the orbit $\mathcal{P}_{n}^{+}$given by (3.77a) with $q=+1$, one obtains that

$$
E\left[L_{n,+; a}\right]=-\frac{n^{2}}{8 \sqrt{a^{2}+1 / 2 \pi}}\left(\frac{3 / 2 \pi+2 a^{2}}{a}\right) .
$$

This approaches $-\infty$ as $a \rightarrow 0$, settling the $\mathcal{P}_{n}^{+}$case. 
The $\mathcal{B}_{n}(b), n \in \mathbf{N}$, case is dealt with by a similar computation using a one parameter curve on this orbit, given by

$$
\begin{aligned}
L_{n, b ; a} & =b^{2}+\frac{n^{2}+4 b^{2}}{2 F_{n, b ; a}}-\frac{3}{4} \frac{n^{2} a^{2}}{F_{n, b ; a}^{2}}, \\
F_{n, b ; a}(x) & =a^{2} \cos ^{2} \frac{n x}{2}+\left(\sin \frac{n x}{2}+\frac{2 b}{n} \cos \frac{n x}{2}\right)^{2},
\end{aligned}
$$

where the parameter $a>0$ is arbitrary. The corresponding solution $\Psi_{a}=\left(\begin{array}{ll}\psi_{1, a} & \psi_{2, a}\end{array}\right)$ of Hill's equation is written as

$$
\begin{aligned}
& \psi_{1, a}(x):=\frac{e^{b x}}{\sqrt{F_{a}(x)}} \sqrt{\frac{n}{2}}\left(\left[\frac{2 b}{n^{2}}+\frac{a^{2}-1}{2 b}\right] \cos \frac{n x}{2}+\frac{2}{n} \sin \frac{n x}{2}\right) \\
& \psi_{2, a}(x):=\frac{e^{-b x}}{\sqrt{F_{a}(x)}} \sqrt{\frac{2}{n}} \cos \frac{n x}{2} .
\end{aligned}
$$

The existence of conformal transformations 'creating' this curve from the 'standard point' in (3.58-59) (which corresponds to $a=1$ ) follows from the fact that (4.35) yields the same monodromy and discrete invariants as (3.58a).

Now it is easy to verify that

$$
E\left[L_{n, b ; a}\right]=b^{2}+\frac{n^{2}+4 b^{2}}{8 a}-\frac{3 n^{2} a}{8},
$$

which is not bounded from below as $a \rightarrow \infty$, completing the proof of the above claim.

\subsection{The exceptional orbit $\mathcal{P}_{1}^{-}$}

In this subsection we prove that on the orbit $\mathcal{P}_{1}^{-}$the chiral energy functional $E[L]$ has the largest lower bound $-1 / 4$, but this value is not reached. The fact that makes $\mathcal{P}_{1}^{-}$exceptional is that for this orbit (and only for this one among the $\mathcal{P}_{n}^{q}, n \neq 0$ ) it is possible to find a number $\xi$ such that

$$
u(\xi)=\frac{1}{2}
$$

and

$$
\psi_{1}(x)>0 \quad \text { for } \quad \xi<x<\xi+2 \pi,
$$

where $u(x)=\psi_{1}(x) / \psi_{2}(x)$, as in Appendix C. Indeed, we can always choose $\xi$ such that (4.37) is satisfied (since the function $u(x)$ takes all values between two consecutive zeros of $\psi_{2}$ ) and for which $\psi_{2}(\xi)$ is positive (since $\psi_{2}$ is antiperiodic). But then (4.38) is also satisfied since $\psi_{1}(\xi)=\frac{1}{2} \psi_{2}(\xi)>0, \psi_{1}(\xi+2 \pi)=u(\xi+2 \pi) \psi_{2}(\xi+2 \pi)=-\frac{1}{2} \psi_{2}(\xi+2 \pi)=$ $\frac{1}{2} \psi_{2}(\xi)=\psi_{1}(\xi)>0$ and $\psi_{1}$ cannot change sign between $\xi$ and $\xi+2 \pi$. The last statement 
holds since a possible zero of $\psi_{1}$ would be a zero of $u(x)$ as well, but we know (see Appendix C) that $u(x)$ increases from $\frac{1}{2}$ to $+\infty$ as $x$ changes from $\xi$ to the singular point of $u(x)$ (the only one in this interval), and then from $-\infty$ to $-\frac{1}{2}$ as $x$ completes its period from the singular point to $\xi+2 \pi$. Our proof is based on the above observation.

We will use the relations

$$
\psi_{1}^{\prime}(\xi+2 \pi)=\psi_{1}^{\prime}(\xi)-\frac{1}{\psi_{1}(\xi)}
$$

and

$$
\int_{\xi}^{\xi+2 \pi} \frac{d x}{\psi_{1}^{2}(x)}=\frac{-1}{u(\xi+2 \pi)}+\frac{1}{u(\xi)}=4,
$$

which also follow from (3.13-14) using the monodromy matrix $P_{\eta}^{q}$ in (3.25a) with $\eta=$ $q=-1$.

As a consequence of the above, the smooth function $y(x):=\psi_{1}(x+\xi+\pi)$ satisfies

$$
y(x)>0 \quad \text { for } \quad x \in[-\pi, \pi], \quad y(\pi)=y(-\pi), \quad y^{\prime}(\pi)=y^{\prime}(-\pi)-\frac{1}{y(\pi)}
$$

and

$$
\int_{-\pi}^{\pi} \frac{d x}{y^{2}(x)}=4
$$

We denote the absolute maximum of $y(x)$ in the interval $[-\pi, \pi]$ by $M$.

Now we express the chiral energy functional (4.1) in terms of $y(x)$ as

$$
E[y]=\frac{1}{2 \pi} \int_{-\pi}^{\pi} d x L(x)=\frac{1}{2 \pi} \int_{-\pi}^{\pi} d x \frac{y^{\prime \prime}}{y}=\frac{1}{2 \pi} \int_{-\pi}^{\pi} d x \frac{y^{\prime 2}}{y^{2}}-\frac{1}{2 \pi y^{2}(\pi)} .
$$

The following consideration is very similar to that of Sect. 4.1., so here we can be brief. We start by integrating the inequality

$$
\frac{1}{2 \pi}\left(\frac{\left|y^{\prime}\right|}{y}-w \sqrt{\frac{M^{2}}{y^{2}}-1}\right)^{2} \geq 0,
$$

where $w$ is a positive constant to be fixed later and obtain

$$
E[y] \geq-\frac{1}{2 \pi y^{2}(\pi)}-w^{2}\left(\frac{2 M^{2}}{\pi}-1\right)+\frac{w}{\pi} \int_{-\pi}^{\pi} d x \frac{\left|y^{\prime}\right|}{y^{2}} \sqrt{M^{2}-y^{2}} .
$$

Then, by an argument similar to that in Sect. 4.1., we can show that

$$
\int_{-\pi}^{\pi} d x \frac{\left|y^{\prime}\right|}{y^{2}} \sqrt{M^{2}-y^{2}} \geq 2 \int_{y(\pi)}^{M} \frac{d y}{y^{2}} \sqrt{M^{2}-y^{2}}=2(\operatorname{tg} p-p),
$$


where equality holds only if $y(x)$ has a single local extremum in the interval $[-\pi, \pi]$ (where it takes its absolute maximum $M$ ) and $p$ is introduced via the parametrization

$$
M=\frac{y(\pi)}{\cos p} \quad 0<p<\frac{\pi}{2}
$$

So far we have shown that for any positive $w$

$$
E[y] \geq \frac{-1}{2 \pi y^{2}(\pi)}-B w^{2}+2 A w
$$

where the positive constants $A, B$ are

$$
A=\frac{1}{\pi}(\operatorname{tg} p-p) \quad B=\frac{2 M^{2}}{\pi}-1
$$

(positivity of $B$ is a consequence of the constraint (4.42)). Choosing $w:=A / B$ yields

$$
E[y] \geq \frac{A^{2}}{B}-\frac{1}{2 \pi y^{2}(\pi)} .
$$

We now parametrize $y(\pi)$ as

$$
y^{2}(\pi)=\frac{\pi}{2} \frac{\sin 2 r}{2 r} \quad 0<r<\frac{\pi}{2} .
$$

This parametrization is not possible if $y^{2}(\pi) \geq \pi / 2$, but in this case $E[y] \geq-1 / \pi^{2}$ manifestly. In this parametrization (4.50) can be written as

$$
E[y] \geq f(p, r)=-\frac{1}{\pi^{2}} \frac{r}{\sin r \cos r}+\frac{r}{\pi^{2}} \frac{(\sin p-p \cos p)^{2}}{\sin r \cos r-r \cos ^{2} p}
$$

It is elementary to show that for $0<p, r<\pi / 2$

$$
f(p, r) \geq f(r, r)=-\frac{1}{\pi^{2}}\left(r^{2}+r \operatorname{ctg} r\right)>-\frac{1}{4}
$$

establishing the strict lower bound $-1 / 4$.

Finally, it is possible to compute the value of the chiral energy functional for the curve (3.77a) on $\mathcal{P}_{1}^{-}$explicitly. We find that $E \rightarrow-1 / 4$ as $a \rightarrow \infty$ along this curve. Thus we have proven that $-1 / 4$ is the largest lower bound but it is not taken on the orbit $\mathcal{P}_{1}^{-}$. This is quite an intriguing orbit since (by (4.1), if $C>0$ ) the corresponding lower bound of the Virasoro zero mode $\mathcal{L}^{0}$ is zero. 


\section{The solutions of the global Liouville equation}

The smooth, periodic solutions of the global Liouville equation (1.1) that we are interested in have two important characteristics. The first is the topological type defined by the number of zeros of $Q(\tau, \sigma)$ for $0 \leq \sigma<4 \pi$ at any fixed $\tau$. This number, $N$, is even on account of the $4 \pi$-periodicity and the fact that $Q$ cannot have a double zero in $\sigma$. The second is the 'Virasoro orbit type' $\mathcal{O}_{+} \times \mathcal{O}_{-}$attached to $Q$ by the orbits $\mathcal{O}_{ \pm}$of the Virasoro densities $L_{ \pm}=\partial_{ \pm}^{2} Q / Q$. This characteristic is a refinement of the monodromy type of the solutions $\Psi_{ \pm}$of (1.3) that compose $Q$ according to (1.5). Correspondingly, we call a solution of (1.1) elliptic, hyperbolic etc if the solutions of the Hill's equations (1.3) have elliptic, hyperbolic etc monodromy. The main purpose of this section is to establish the precise relationship between the two characteristics.

We shall make use of the normal forms of Hill's equation to write down explicit representatives for all smooth, periodic solutions of (1.1) up to conformal transformations. As explained in the Introduction, these representatives can be obtained by taking $\Psi_{+}$ in (1.5) to run through the representative solutions given in Section 3, and choosing $\Psi_{-}$ from an analogous set of representatives in such a way to obey the monodromy constraint (1.7), which ensures the periodicity of $Q$. Inspection of the representative solutions will then reveal the relation between the topological type and the Virasoro orbit type. The results in Section 4 will further permit to describe the behaviour of the energy functional in the various sectors of the global Liouville model. According to eqs. (2.22-23), the (total) energy of a solution $Q$ is given by

$$
\mathcal{H}_{\mathrm{WZ}}[Q]=2 \pi \kappa\left(E\left[L_{+}\right]+E\left[L_{-}\right]\right),
$$

where $E\left[L_{ \pm}\right]$is the chiral energy at the respective Virasoro density $L_{ \pm}$. The energy is bounded from below on the conformal orbit $\left\{Q^{\alpha_{+}, \alpha_{-}}\right\}$of a solution $Q$ (see (1.9)) if and only if the corresponding chiral energy functionals $E_{L_{ \pm}}\left[\alpha_{ \pm}\right]$are both bounded from below and $\kappa>0$. Hence, in the rest of this section we assume that $\kappa>0$.

Below, the number of zeros of $Q$ turns out to be a function of the discrete parameters of the Virasoro orbits. For elliptic orbits $\mathcal{C}\left(\nu_{+}\right) \times \mathcal{C}\left(\nu_{-}\right)$, we find that $N=\left(\nu_{+}+\nu_{-}\right)$, where the sum only depends on the discrete parameters $d_{ \pm}$attached to $\nu_{ \pm}$by (3.33). For $\mathcal{E}_{n}$ type orbits the same formula is valid in the limiting case of integral $\nu_{ \pm}$. Actually, the formula $N=\left(\nu_{+}+\nu_{-}\right)$can be used to describe most other cases, too, if we deform the representatives of the Virasoro orbits according to Figure 1 and note that as a topological invariant $N$ cannot depend on such deformations. (It is for the same reason that $N$ cannot depend on the continuous parameters of the monodromy.) Consistently with the earlier results $[15,16]$, we shall see that the $N=0$ sector, which contains the regular solutions of the Liouville equation (1.2), is associated with the Virasoro orbits $\mathcal{B}_{0}(b) \times \mathcal{B}_{0}(b)$, and it turns out that the energy is bounded from below in this sector only. 
In Ref. [3] it was argued that the $\mathrm{N}=2$ sector is special in that it contains a stable lowest energy state (the one with constant Virasoro densities) and is therefore a suitable starting point for quantization. As we show in Appendix $\mathrm{D}$, the $\mathrm{N}=2$ sector is connected and the would-be vacuum state of Ref. [3] (which in our notation is given by eq. (5.8) below) is only locally stable since it is continuously connected to states whose energy is not bounded from below.

\subsection{Elliptic solutions}

If $Q$ is a solution so that $\Psi_{+}$in (1.5) has elliptic monodromy, then using the freedom (1.8) we may assume that $M_{\Psi_{+}}$is of the standard form (3.23a) with some $\omega:=\omega_{+}$,

$$
M_{\Psi_{+}}=C\left(\omega_{+}\right), \quad 0<\omega_{+}<2 \pi, \quad \omega_{+} \neq \pi
$$

Then (1.7) forces

$$
M_{\Psi_{-}}=C\left(\omega_{-}\right) \quad \text { with } \quad \omega_{-}=2 \pi-\omega_{+} .
$$

Therefore the parameters $\nu_{ \pm}$of the respective chiral and anti-chiral Virasoro orbits $\mathcal{C}\left(\nu_{ \pm}\right)$ take the form

$$
\nu_{+}=\frac{\omega_{+}}{\pi}+2 d_{+}, \quad \nu_{-}=\left(2-\frac{\omega_{+}}{\pi}\right)+2 d_{-}, \quad d_{ \pm} \in \mathbf{Z}_{+},
$$

which fixes the the allowed pairing

$$
\mathcal{C}\left(\nu_{+}\right) \times \mathcal{C}\left(\nu_{-}\right)
$$

From the results of Section 3.3, a representative solution $Q$ with this Virasoro orbit type can be given explicitly as

$$
Q\left(x^{+}, x^{-}\right)=\frac{2}{\sqrt{\nu_{+} \nu_{-}}} \cos \theta
$$

where

$$
\theta=\frac{1}{2}\left(\nu_{+} x^{+}-\nu_{-} x^{-}\right)=\frac{1}{2}\left(\frac{\omega_{+}}{\pi}+d_{+}-d_{-}-1\right) \tau+\frac{1}{2}\left(d_{+}+d_{-}+1\right) \sigma .
$$

The Virasoro densities belonging to this solution are $L_{ \pm}=-\frac{1}{4} \nu_{ \pm}^{2}$, and $Q^{\alpha_{+}, \alpha_{-}}$in (1.9) is the general solution associated with elliptic monodromy. The number of zeros of such a solution over a $4 \pi$ period in $\sigma$ is

$$
N=2\left(d_{+}+d_{-}+1\right)=\nu_{+}+\nu_{-} .
$$

In particular, $N \neq 0$.

On the orbit of the conformal group $\widetilde{\operatorname{Diff}}_{0}\left(S^{1}\right) \times \widetilde{\operatorname{Diff}}_{0}\left(S^{1}\right)$ through $Q$ in (5.4) the energy is not bounded from below. This follows from the results in the preceding section since either $\nu_{+}$or $\nu_{-}$is greater than 1 by $(5.3 \mathrm{a})$. 


\subsection{Solutions associated with monodromy type $E_{\eta}$}

By (1.7), the list of possible Virasoro orbit types is in this case

$$
\mathcal{E}_{n_{+}} \times \mathcal{E}_{n_{-}} \quad \text { with } \quad n_{ \pm} \in \mathbf{N}, \quad\left(n_{+}+n_{-}\right) \in 2 \mathbf{N} .
$$

For any such orbit, there is a unique solution of (1.1) up to conformal transformations:

$$
Q\left(x^{+}, x^{-}\right)=\frac{2}{\sqrt{n_{+} n_{-}}} \cos \frac{\left(n_{+} x^{+}-n_{-} x^{-}\right)}{2},
$$

whose topological type is given by $N=\left(n_{+}+n_{-}\right)$. Of particular interest is the $N=2$ representative solution,

$$
Q\left(x^{+}, x^{-}\right)=2 \cos \left(\frac{1}{2} \sigma\right) .
$$

This is the only one among the $E_{\eta}$-type solutions such that on the orbit $\left\{Q^{\alpha_{+}, \alpha_{-}}\right\}$of $\widetilde{\operatorname{Diff}}_{0}\left(S^{1}\right) \times \widetilde{\operatorname{Diff}}_{0}\left(S^{1}\right)$ the energy is bounded from below. The static solution $Q$ in (5.8) may be thought of as the classical analogue of a vacuum state in a conformal field theory, since the Virasoro zero modes $\mathcal{L}_{ \pm}^{0}(4.1)$ are equal to zero at $Q$ and are bounded from below on the associated coadjoint orbits. However, unlike in standard conformal field theory, the energy is not bounded from below in the global Liouville model (1.1).

\subsection{Hyperbolic solutions}

When constructing the hyperbolic solutions of (1.1) by (1.5), we may assume that the chiral monodromy matrix $M_{\Psi_{+}}$is of the standard form $B_{\eta}(b)$ in (3.22a), and then (1.7) requires the anti-chiral monodromy matrix $M_{\Psi_{-}}$to coincide with the chiral one. This means that the set of hyperbolic solutions is a union over the Virasoro orbit types

$$
\mathcal{B}_{n_{+}}(b) \times \mathcal{B}_{n_{-}}(b) \quad \text { where } \quad b>0, \quad(-1)^{n_{+}+n_{-}}=1 .
$$

We shall verify that the topological type of these solutions is given by

$$
N=\left(n_{+}+n_{-}\right) .
$$

On a set of solutions whose Virasoro orbit type involves $\mathcal{B}_{n}(b)$ for some $n>0$, the energy is not bounded from below. Next we elaborate the simplest case $n_{ \pm}=0$, for which the energy is bounded from below.

5.3.1. Solutions with Virasoro orbit type $\mathcal{B}_{0}(b) \times \mathcal{B}_{0}(b)$. For fixed $b>0$, the conformally nonequivalent solutions of Hill's equation are given by (3.55). Applying this to the chiral and anti-chiral equations in (1.3), we obtain from (1.5) the following two conformally nonequivalent solutions of the global Liouville equation:

$$
Q_{ \pm}\left(x^{+}, x^{-}\right)= \pm \frac{1}{b} \cosh b\left(x^{+}+x^{-}\right)= \pm \frac{1}{b} \cosh b \tau .
$$


As they are $\sigma$ independent, these solutions have no zeros, $N=0$. We shall see that all other Virasoro orbit types belong to $N \neq 0$.

The global Liouville equation (1.1) is invariant under $Q \mapsto-Q$, and the topological sector $N=0$ further decomposes into two disconnected subsectors according to the sign of $Q$. Clearly, both these subsectors correspond to the regular solutions of the Liouville equation(1.2) by the substitutions $Q= \pm e^{-\frac{1}{2} \varphi}$.

It is interesting to recover the standard form of the solutions of (1.2) from $Q_{+}^{\alpha_{+}, \alpha_{-}}$ with $Q_{+}$in (5.11), which yields

$$
e^{-\frac{1}{2} \varphi\left(x^{+}, x^{-}\right)}=\frac{1}{b \sqrt{\partial_{+} \alpha_{+}\left(x^{+}\right) \partial_{-} \alpha_{-}\left(x^{-}\right)}} \cosh b\left(\alpha_{+}\left(x^{+}\right)+\alpha_{-}\left(x^{-}\right)\right),
$$

where $\alpha_{+}, \alpha_{-}$now satisfy (1.10), ensuring the periodicity and the smoothness of $\varphi$. This can be rewritten in Liouville's form [17]

$$
\varphi\left(x^{+}, x^{-}\right)=\ln \left(\frac{\partial_{+} A\left(x^{+}\right) \partial_{-} A_{-}\left(x^{-}\right)}{\left(A_{-}\left(x^{-}\right)-A_{+}\left(x^{+}\right)\right)^{2}}\right)
$$

by putting

$$
A_{+}\left(x^{+}\right):=-e^{-2 b \alpha_{+}\left(x^{+}\right)}, \quad A_{-}\left(x^{-}\right):=e^{2 b \alpha_{-}\left(x^{-}\right)} .
$$

Of course, if we 'forget' these definitions of $A_{ \pm}$, then we could also describe the singular solutions of (1.2) by (5.13). However, it appears more economical and transparent to do so in terms of the variable $Q$ by means of our group theoretic analysis.

5.3.2. Solutions of type $\mathcal{B}_{0}(b) \times \mathcal{B}_{2 n}(b)$ or $\mathcal{B}_{2 n}(b) \times \mathcal{B}_{0}(b)$ for $n \in \mathbf{N}$. By the analysis in Section 3.5, one needs a singe representative solution $Q$ of type $\mathcal{B}_{0}(b) \times \mathcal{B}_{2 n}(b)$, e.g.,

$$
Q\left(x^{+}, x^{-}\right)=\frac{1}{\sqrt{2 n b F_{2 n, b}\left(x^{-}\right)}}\left\{e^{-b \tau} \cos n x^{-}+e^{b \tau}\left(\sin n x^{-}+\frac{b}{2 n} \cos n x^{-}\right)\right\},
$$

where $F_{2 n, b}$ is defined in (3.58). It follows that $\left.Q\right|_{\tau=0}=0$ if and only if $\operatorname{tg} \frac{n \sigma}{2}=\frac{n+b}{n}$, which implies that $N=2 n$. In the case $\mathcal{B}_{2 n}(b) \times \mathcal{B}_{0}(b)$, a representative solution is obtained from (5.15) using the symmetry of the global Liouville equation under the interchange of the light cone coordinates, whereby $Q \mapsto \tilde{Q}$ with $\tilde{Q}\left(x^{+}, x^{-}\right):=Q\left(x^{-}, x^{+}\right)$.

5.3.3. Virasoro orbit type $\mathcal{B}_{n_{+}}(b) \times \mathcal{B}_{n_{-}}(b)$ for $n_{+} n_{-} \neq 0$. Using the solution (3.58) of Hill's equation, we can now write down the representative solution of the global Liouville equation as

$$
\begin{gathered}
Q\left(x^{+}, x^{-}\right)=2 \frac{e^{-b \tau} \cos \frac{n_{+} x^{+}}{2} \cos \frac{n_{-} x^{-}}{2}+e^{-b \tau} X}{\sqrt{n_{+} n_{-} F_{n_{+}, b}\left(x^{+}\right) F_{n_{-}, b}\left(x^{-}\right)}} \\
X=\left(\sin \frac{n_{+} x^{+}}{2}+\frac{b}{n_{+}} \cos \frac{n_{+} x^{+}}{2}\right)\left(\sin \frac{n_{-} x^{-}}{2}+\frac{b}{n_{-}} \cos \frac{n_{-} x^{-}}{2}\right) .
\end{gathered}
$$


Either directly comparing (5.7) and (5.16), or by recalling a remark after (3.59), we see that this hyperbolic solution is a smooth deformation of the $\mathcal{E}_{n_{+}} \times \mathcal{E}_{n_{-}}$type solution (5.7), to which it reduces in the $b=0$ limit. Therefore $Q$ in (5.16) has the same number of zeros as $Q$ in (5.7), namely, $N=\left(n_{+}+n_{-}\right) \in 2 \mathbf{N}$.

\subsection{Parabolic solutions}

The representatives $P_{\eta}^{q}$ of the parabolic conjugacy classes in (3.25a) obey

$$
\left(P_{\eta}^{q}\right)^{T}=w^{-1} P_{\eta}^{-q} w \quad \text { where } \quad w:=\left(\begin{array}{cc}
0 & -1 \\
1 & 0
\end{array}\right)
$$

Hence in the parabolic case the periodic solutions of (1.1) have Virasoro orbit types

$$
\mathcal{P}_{n_{+}}^{q} \times \mathcal{P}_{n_{-}}^{-q}, \quad n_{ \pm} \in \mathbf{Z}_{+}, \quad\left(n_{+}+n_{-}\right) \in 2 \mathbf{N}
$$

If we choose $M_{\Psi_{+}}=P_{\eta}^{q}\left(\eta=(-1)^{n_{+}}\right)$in the construction $(1.5)$, then $M_{\Psi_{-}}$has to be chosen as $M_{\Psi_{-}}=w^{-1} P_{\eta}^{-q} w$. The solutions of Hill's equation with monodromy $M_{\Psi_{-}}$ can be obtained from those in Section 3.6 by means of a transformation like in (3.15). Observe that the energy is not bounded from below in correspondence with any of the Virasoro orbit types in (5.18). It turns out that the topological type of the solutions is given by $N=\left(n_{+}+n_{-}\right)$similar to the hyperbolic case (5.10).

5.4.1. Solutions of type $\mathcal{P}_{0}^{+} \times \mathcal{P}_{2 n}^{-}$or $\mathcal{P}_{2 n}^{-} \times \mathcal{P}_{0}^{+}$for $n \in \mathbf{N}$. It is clear from the study of Hill's equation in Section 3.6 that we may choose a single representative solution of type $\mathcal{P}_{0}^{+} \times \mathcal{P}_{2 n}^{-}$, for example,

$$
Q\left(x^{+}, x^{-}\right)=\sqrt{\frac{2 \pi}{H_{2 n,-}\left(x^{-}\right)}}\left(\frac{\tau}{2 \pi} \sin n x^{-}+\frac{1}{n} \cos n x^{-}\right)
$$

with $H_{2 n,-}$ defined in (3.71b). Setting $\tau=0$, we see that the number of zeros of $Q$ is $2 n$. We can use the symmetry of (1.1) under $x^{ \pm} \mapsto x^{\mp}$ to obtain a solution of type $\mathcal{P}_{2 n}^{-} \times \mathcal{P}_{0}^{+}$.

5.4.2. Virasoro orbit type $\mathcal{P}_{n_{+}}^{q} \times \mathcal{P}_{n_{-}}^{-q}$ for $n_{+} n_{-} \neq 0$. Up to conformal transformations, the corresponding solution is given as follows:

$$
Q\left(x^{+}, x^{-}\right)=\frac{\left(\frac{2}{n_{-}} \sin \frac{n_{+} x^{+}}{2} \cos \frac{n_{-} x^{-}}{2}+\frac{q \tau}{2 \pi} \sin \frac{n_{+} x^{+}}{2} \sin \frac{n_{-} x^{-}}{2}-\frac{2}{n_{+}} \cos \frac{n_{+} x^{+}}{2} \sin \frac{n_{-} x^{-}}{2}\right)}{\sqrt{H_{n_{+}, q}\left(x^{+}\right) H_{n_{-}, q}\left(x^{-}\right)}} .
$$

At $\tau=0$, this simplifies to the product of a factor without zeros and

$$
Y(\sigma):=2 n_{-} \sin \frac{n_{-} \sigma}{4} \cos \frac{n_{+} \sigma}{4}+2 n_{+} \sin \frac{n_{+} \sigma}{4} \cos \frac{n_{+} \sigma}{4} .
$$


One can check that $Y(\sigma)$ has $\left(n_{+}+n_{-}\right)$zeros for $0 \leq \sigma<4 \pi$.

Alternatively, one may prove the relation $N=\left(n_{+}+n_{-}\right)$using the curve of parabolic solutions of Hill's equation given in (3.77), which become the $\mathcal{E}_{n}$ representatives in the $a \rightarrow \infty$ limit. Noting that the topological type of the corresponding $a$-dependent family of solutions of (1.1) is constant, one obtains $N=\left(n_{+}+n_{-}\right)$for the parabolic solutions of (1.1) as a consequence of the analogous relation in the $a \rightarrow \infty$ limit.

To summarize, we enumerated the conformally nonequivalent, smooth, periodic solutions of the global Liouville equation, and established the dependence of the topological type $N$ on the discrete parameters of the Virasoro coadjoint orbits. Combining these results with the analysis of Section 4 , we may conclude that the energy $\mathcal{H}_{\mathrm{Wz}}[Q]$ in (5.1) (with $\kappa>0$ ) is bounded from below only in the $N=0$ topological sector of the model. For instance, if $N=2$ then we have solutions with Virasoro orbit type $\mathcal{E}_{1} \times \mathcal{E}_{1}$ on which the energy is bounded from below, and also of type $\mathcal{B}_{1}(b) \times \mathcal{B}_{1}(b)$, as well as others, on which the energy is unbounded. If $N=0$ then the sign of $Q$ is a further topological invariant, but otherwise any two solutions with the same number of zeros can be deformed into each other. This can be seen with the aid of our explicit results on the solutions, or can be established by directly inspecting the phase space of the global Liouville model, the details are collected in Appendix D.

For completeness, let us finally comment on the present understanding of the role of the various classical solutions in the quantized Liouville field theory. First, the quantizations of Thorn et al [18] and Otto and Weigt [19] deal with the regular sector, which contains a subset of the hyperbolic solutions. Second, the studies of Gervais and collaborators (see e.g. [20]) deal with the elliptic sector of the theory. More general singular solutions appear in the work [3], but see our remark at the end of the introduction to Section 5 .

\section{Discussion}

In this paper we dealt with the coadjoint orbits of the Virasoro algebra and the solution space of the global Liouville equation. Our description of the Virasoro orbits is summarized by Figure 1. We connected our elementary presentation with the well-known classifications of the Virasoro orbits [8,9,11], whereby we hope to have made the known results more easily accessible, and we also made them more explicit and more complete in some respects. Our review of the orbits was motivated by its subsequent application to the global Liouville equation (1.1). The main interest of this equation is that its smooth solutions describe also those solutions of the Liouville equation (1.2) that may be singular in terms of $\varphi$ in such a way that the Virasoro densities $L_{ \pm}$, the components of the conformally improved Liouville stress-energy tensor, remain smooth. We have listed

all smooth solutions of the global Liouville equation up to conformal transformations 
under periodic boundary condition. In this way we established the correlation between the topological type (given by the number of zeros of $Q$ ) of the solutions and their Virasoro orbit type. The energy (5.1) (with $\kappa>0$ ) turned out to be bounded from below only in the trivial topological sector that contains the solutions with Virasoro orbit type $\mathcal{B}_{0}(b) \times \mathcal{B}_{0}(b)$.

The antiperiodic, $Q\left(x^{+}+2 \pi, x^{-}-2 \pi\right)=-Q\left(x^{+}, x^{-}\right)$, solutions of (1.1) can be analyzed analogously to the periodic case, since $L_{ \pm}$are periodic for these solutions, too. In the antiperiodic case $Q$ has an odd number of zeros for $0 \leq \sigma<4 \pi$, and the possible pairings of the chiral and antichiral Virasoro densities are governed by the monodromy condition $M_{\Psi_{-}}=-\left(M_{\Psi_{+}}\right)^{T}$ that replaces (1.7). Similar to (5.5), we still have $N=\left(\nu_{+}+\nu_{-}\right)$for elliptic solutions. An interesting consequence is that in the $N=1$ sector we can pair the orbits as $\mathcal{C}\left(\nu_{+}\right) \times \mathcal{C}\left(\nu_{-}\right)$for $0<\nu_{+}<1, \nu_{-}=\left(1-\nu_{+}\right)$, and therefore the energy is bounded from below on the set of the corresponding solutions. However, taking the other monodromies into account, the energy is not bounded from below in any of the topological sectors in the antiperiodic case (cf. Ref. [3]). For instance, if $N=1$ then the Virasoro orbit type $\mathcal{B}_{0}(b) \times \mathcal{B}_{1}(b)$, on which the energy is unbounded, is also permitted, and any two solutions with the same $N$ can be connected to each other along a path of solutions.

Throughout this paper we required that the Virasoro densities $L_{ \pm}$, and therefore also the global Liouville field $Q$ and the conformal transformations $\alpha_{ \pm} \in \widetilde{\operatorname{Diff}}_{0}\left(S^{1}\right)$, be given by infinitely many times differentiable functions of $x^{ \pm}$. From the point of view of the differential equations (1.1) and (1.3), it might be natural to relax this stringent requirement and assume instead that the conformal transformations and the global Liouville field are thrice and twice continuously differentiable functions, respectively, while the Virasoro densities are assumed to be only continuous. Under these requirements the set of possible Virasoro densities becomes larger, but so does the group of conformal transformations too. As a result, the classification of the Hill's equations in Section 3 and the solutions of the global Liouville equation in Section 5, including the list of nonequivalent representatives given in the respective sections, remain valid without change.

The previous studies [2,3] of the singular solutions of the Liouville equation were already mentioned in the Introduction. In Ref [2], whose analytic methods are rather different from our group theoretic approach, the admissible singularities of the field $\varphi$ and the boundary conditions at infinity are specified directly in such a way that their nature is preserved by the time development, and the regularity of the stress-energy tensor as well as the existence of certain associated Noether charges are ensured. For any fixed solution $\varphi(\tau, \sigma)$, the time development of the singularities is then interpreted [2] as the dynamics of certain 'particles' on the line interacting with each other through a velocity dependent potential. For some particular solutions, the dynamics of the singularities is 
even Poincaré invariant. It could be interesting to generalize this picture and describe the dynamics of the zeros of our smooth and periodic $Q$ subject to (1.1) in terms of some system of interacting particles on the circle. Some results in Ref. [21] may be related to this problem.

As we saw in Section 2, the global Liouville system is a reduced WZNW model [4] for the group $S L(2, \mathbf{R})$. For other groups, the reduced WZNW systems [4] contain the standard Toda field theories in their trivial topological sector. Some nontrivial aspects of these reduced systems have been investigated in the point particle case $[6,22,23,24]$. In the field theoretic case the $S L(3, \mathbf{R})$ model was studied [25] using the known description of the symplectic leaves of the $W_{3}$-algebra [26] that replaces the Virasoro algebra as one goes from $S L(2, \mathbf{R})$ to $S L(3, \mathbf{R})$. However, the results are far from complete even in this particular case, and exploring the solution space of the 'global Toda models' is a problem for the future.

Finally, the most interesting problem is of course to better understand the role of the singular solutions of the Liouville and Toda equations at the quantum mechanical level. As was already mentioned, results in this direction can be found in Ref. [3] and in the works [20]. However, a full treatment including all types of singular solutions appears to be still missing.

Acknowledgements. L.F. wishes to thank I. Tsutsui for discussions and is grateful to the Alexander von Humboldt Foundation for a fellowship. This work was supported in part by the Hungarian National Science Fund (OTKA) under T016251 and T019917.

\section{Appendix A: The number of zeros of $Q$ as a winding number}

In this appendix we study the connection between the number of zeros $N[Q]$ of the field $Q$, and the winding number $W[g]$ of the corresponding $S L(2, \mathbf{R})$ valued WZNW field $g$. Both quantities are topological invariants, so it is natural to assume that they are related, and we shall find that the relationship is given by eq. (A.6) below.

We need a concrete description of the winding number associated with the WZNW field $g(\sigma) \in S L(2, \mathbf{R})$. For this we parametrize the $S L(2, \mathbf{R})$ matrix elements

$$
g=\left(\begin{array}{ll}
a & b \\
c & d
\end{array}\right) \quad a d-b c=1
$$

using the three-dimensional system of coordinates $(\phi, x, y)$ as

$$
\begin{array}{ll}
d-a=2 x & d+a=2 r \sin \phi, \\
b+c=2 y & b-c=2 r \cos \phi,
\end{array}
$$


where $r=\sqrt{1+x^{2}+y^{2}}$. We introduced this system of coordinates because it makes transparent that the group $S L(2, \mathbf{R})$ has the topology of $U(1) \times \mathbf{R}^{2}$. The winding number of the WZNW field $g(\sigma)$ is the number of times $\exp (i \phi(\sigma))$ goes around the $U(1)$ circle as the field completes a full period $4 \pi$ in $\sigma$. It may be determined as the regular integral

$$
W[g]=\frac{1}{2 \pi} \int_{0}^{4 \pi} d \sigma \frac{(b-c)\left(a^{\prime}+d^{\prime}\right)+\left(c^{\prime}-b^{\prime}\right)(a+d)}{4+(a-d)^{2}+(b+c)^{2}}
$$

but it is more useful to determine it directly as $\frac{1}{2 \pi}$-times the total change in the angle

$$
\phi(\sigma)=\operatorname{arctg}\left(\frac{a+d}{b-c}\right)(\sigma)
$$

as $\sigma$ varies over a $4 \pi$-period.

Now we restrict the WZNW field $g(\sigma)$ to the gauge in (2.8), where by $(2.9)$ and (2.10) we have

$$
\begin{aligned}
& a+d=\ell_{+} g_{22}-2 \kappa P_{+}^{\prime}+g_{22}=g_{22}+\frac{1}{g_{22}}\left(1+P_{+}^{2}-2 \kappa P_{+} g_{22}^{\prime}\right), \\
& b-c=2 \kappa g_{22}^{\prime}, \quad\left(Q=\kappa g_{22}\right) .
\end{aligned}
$$

This shows that for the gauge fixed configuration $(a+b) /(b-c)$ in (A.4) is singular precisely at the points where $Q^{\prime}$ vanishes, and that the sign of the argument of the arctg function close to a point where it is singular is equal to $\operatorname{sign}(\kappa) \operatorname{sign}(Q) \operatorname{sign}\left(Q^{\prime}\right)$. Clearly, the contribution to the winding number of an interval between two consecutive zeros of $Q^{\prime}$ depends only on the product of these signs at the beginning and at the end of the interval. Since the sign of $Q^{\prime}$ does not change within such an interval, there is no contribution coming from the interval if the sign of $Q$ does not change either, as for example between points 1 and 2 or 2 and 3 on Figure 3. 


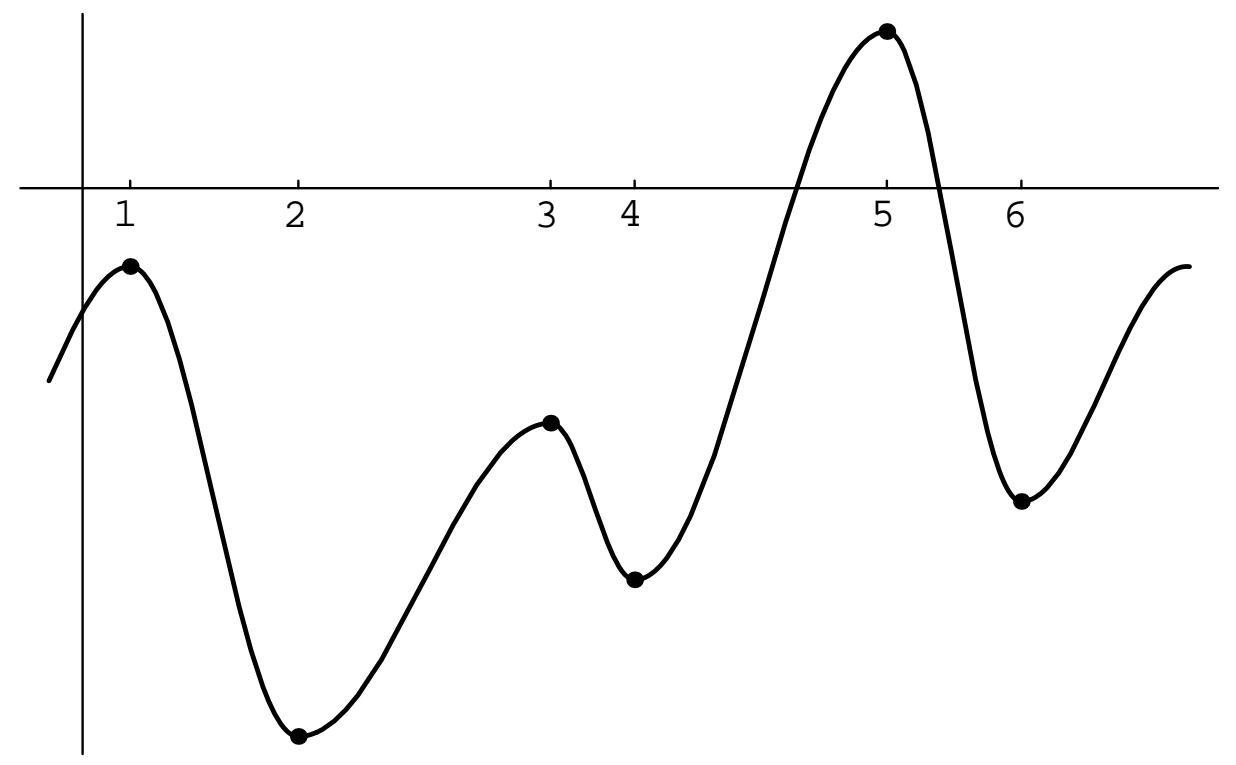

Figure 3: An example of the function $Q(\sigma)$

There is a nonvanishing contribution to $W$ only if $Q$ changes sign between two consecutive zeros of $Q^{\prime}$. This happens if the interval contains a zero of $Q$, and in such a case the contribution is always $\operatorname{sign}(\kappa) / 2$, independently of whether the curve reaches the zero from below or from above. (See the interval between points 4 and 5 and the interval between 5 and 6 on Figure 3.) It follows from the above observations that the winding number of a WZNW field $g$ in (2.8) corresponding to a global Liouville field $Q=\kappa g_{22}$ with $2 n$ zeros is $\operatorname{sign}(\kappa) n$. That is we have

$$
N[Q]=2 \operatorname{sign}(\kappa) W[g]
$$

for any configuration in (2.8). This means in particular that the constraints in (2.6) exclude those WZNW configurations with nonzero winding number for which the sign of the winding number does not agree with the sign of $\kappa$.

\section{Appendix B: The little groups for orbits with constant Virasoro densities}

We below investigate the little groups for the orbits that contain a constant Virasoro density, $L=\Lambda \in \mathbf{R}$, by integrating the equation

$$
\Lambda=\alpha^{\prime 2} \Lambda+S(\alpha) .
$$


Like in Subsection 4.1, it proves useful to introduce the function

$$
q=\frac{1}{\left(\alpha^{-1}\right)^{\prime}}=\alpha^{\prime} \circ \alpha^{-1}
$$

Recall from (4.9) that $q$ is smooth, $2 \pi$-periodic and positive.

We consider first the orbits with elliptic monodromy, for which $\Lambda=-\frac{\nu^{2}}{4}$. In this case (B.1) reads in terms of $q$ as

$$
-\frac{\nu^{2}}{4}=-\frac{\nu^{2}}{4} q^{2}-\frac{1}{2} q q^{\prime \prime}+\frac{1}{4}\left(q^{\prime}\right)^{2} .
$$

By the substitution $q=f^{2} / 2$, this simplifies to a problem of classical mechanics in one dimension:

$$
f^{\prime \prime}=\nu^{2}\left(\frac{1}{f^{3}}-\frac{1}{4} f\right),
$$

which can be solved by quadrature. The solution will be real if the constant of integration, $a$, arising in the first integration,

$$
\frac{1}{2}\left(f^{\prime}\right)^{2}=a-\frac{\nu^{2}}{2}\left(\frac{1}{f^{2}}+\frac{f^{2}}{4}\right)
$$

satisfies $a \geq \frac{\nu^{2}}{2}$. In this case the second integration yields

$$
q=\frac{2 a}{\nu^{2}}+\sqrt{\left(\frac{2 a}{\nu^{2}}\right)^{2}-1} \sin (\nu x+c)
$$

where $c$ is the corresponding constant of integration. The function $q$ given by (B.6) is positive for all values of $a$ and $\nu$, and it is $2 \pi$-periodic if and only if

$$
\text { (i) } \quad \nu \notin \mathbf{N}, a=\frac{\nu^{2}}{2} ; \quad \text { (ii) } \quad \nu \in \mathbf{N}, a \geq \frac{\nu^{2}}{2} .
$$

In case (i) one easily gets from the definition of $q$ that $\alpha(x)=x+\alpha_{0}$, describing the lift of the group of rigid rotations $\operatorname{Rot}\left(S^{1}\right)=S^{1}$ to the translation group of $\mathbf{R}$. In case (ii), which corresponds to orbits with $E_{\eta}$-type monodromy, we find from inverting the relation between $q$ and $\alpha$ that

$$
\alpha(x)=\frac{2}{n} \operatorname{arctg}\left(\frac{\operatorname{tg}\left(\frac{n}{2} x+\hat{c}\right)}{\frac{2 a}{n^{2}}+\sqrt{\left(\frac{2 a}{n^{2}}\right)^{2}-1}}\right)+\alpha_{0},
$$

where $\hat{c}$ is a new constant of integration, and $\alpha_{0}$ is a constant related to $c$. To exhibit the $S L(2, \mathbf{R})(S U(1,1))$ character of these conformal transformations we use the well-known 
relation between the inverse trigonometric and logarithmic functions, which allows us to rewrite (B.7) as

$$
\alpha(x)=\frac{1}{i n} \ln \frac{A e^{i n x}+\bar{B}}{B e^{i n x}+\bar{A}},
$$

where

$$
A=\frac{M+1}{2 \sqrt{M}} e^{i\left(2 \hat{c}+\alpha_{0} n\right) / 2}, \quad \bar{B}=\frac{M-1}{2 \sqrt{M}} e^{-i\left(2 \hat{c}-\alpha_{0} n\right) / 2}, \quad M=\frac{2 a}{n^{2}}+\sqrt{\left(\frac{2 a}{n^{2}}\right)^{2}-1} .
$$

These equations show that $\chi(\alpha) \in \operatorname{Diff}_{0}\left(S^{1}\right)$, see eq. (3.4), operates on $z=e^{i x} \in S^{1}$ according to

$$
\chi(\alpha): z \mapsto\left[\frac{A z^{n}+\bar{B}}{B z^{n}+\bar{A}}\right]^{\frac{1}{n}} \quad \text { where } \quad\left(\begin{array}{cc}
A & \bar{B} \\
B & \bar{A}
\end{array}\right) \in S U(1,1) .
$$

Therefore, for any $n \in \mathbf{N}$, the little group of $L=-\frac{n^{2}}{4}$ in $\operatorname{Diff}_{0}\left(S^{1}\right)$ is an $n$-fold cover of the Möbius group $P S L(2, \mathbf{R})$.

In the case of orbits with hyperbolic monodromy, for which $\Lambda=b^{2}$ with $b>0$ as in (3.52), the integration of the equation replacing (B.4),

$$
f^{\prime \prime}=b^{2}\left(f-\frac{4}{f^{3}}\right)
$$

yields

$$
\frac{1}{2}\left(f^{\prime}\right)^{2}=a+b^{2}\left(\frac{2}{f^{2}}+\frac{f^{2}}{2}\right)
$$

instead of (B.5). The constant $a$ must satisfy $a \geq-2 b^{2}$ to guarantee the positivity of the r.h.s. Since the integration of eq. (B.8) involves hyperbolic functions rather than trigonometric ones, it is not difficult to see that the only periodic solution is $f^{2} \equiv 2$, which is obtained for $a=-2 b^{2}$. This solution, obviously, leads again to $\alpha(x)=x+\alpha_{0}$.

Among the orbits with parabolic monodromy only $\mathcal{P}_{0}^{+}$has a constant Virasoro density, and this corresponds to $\Lambda=0$. In this case eq. (B.4) simplifies to $f^{\prime \prime}=0$, which is solved by $f=a x+c$. This solution is periodic only for $a=0$, and with this choice (after tuning the constant of integration $c$ as required by eq. (1.10)) it leads again to $\alpha(x)$ describing the lift of the group of rigid rotations.

\section{Appendix C: Construction of conformal transformations for $\mathcal{B}_{n}(b)$ and $\mathcal{P}_{n}^{q}$}

We here show that any two solution vectors, say $\Psi=\left(\begin{array}{ll}\psi_{1} & \psi_{2}\end{array}\right)$ and $\bar{\Psi}=\left(\begin{array}{ll}\bar{\psi}_{1} & \bar{\psi}_{2}\end{array}\right)$, of Hill's equation that have the same monodromy matrix $B_{\eta}(b)$ (or $P_{\eta}^{q}$ ) and discrete invariant $n>0$ (3.43) can be transformed into each other by a conformal transformation. 
In particular, this proves that $\Psi$ in (3.58) (and that in (3.71)) is indeed a representative for the solutions associated with the Virasoro orbit $\mathcal{B}_{n}(b)$ (and respectively with $\mathcal{P}_{n}^{q}$ ).

For definiteness, let us concentrate on the case $\mathcal{B}_{n}(b)$. Denote the zeros of the component $\psi_{2}$ (the number of which, within a $2 \pi$ period, is $n$ ) by $x_{k}$ and arrange the indexing of these zeros so that

$$
\begin{aligned}
0 & \leq x_{1}<x_{2} \ldots x_{n}<2 \pi, \\
x_{k+m n} & =x_{k}+2 m \pi, \quad k=1, \ldots, n \quad m \in \mathbf{Z} .
\end{aligned}
$$

Because of the Wronskian condition, the derivative of $\psi_{2}$ cannot vanish at the points $x_{k}$ and we have

$$
\begin{aligned}
& \psi_{2}^{\prime}\left(x_{k}\right)=r_{k} \neq 0, \\
& \psi_{1}\left(x_{k}\right)=\frac{-1}{r_{k}} .
\end{aligned}
$$

The ratio

$$
u(x)=\frac{\psi_{1}(x)}{\psi_{2}(x)} \quad x \neq x_{k}
$$

is well-defined and smooth away from the set of zeros and has the properties

$$
\begin{aligned}
u(x+2 \pi) & =e^{4 \pi b} u(x), \\
u^{\prime}(x) & =\frac{1}{\psi_{2}^{2}(x)}>0 .
\end{aligned}
$$

Near the point $x_{k} u(x)$ can be written as

$$
u(x)=\frac{-1}{\left(x-x_{k}\right) r_{k}^{2}}+\beta_{k}(x),
$$

where $\beta_{k}(x)$ is smooth around $x_{k}$. Using (C.4) and (C.5) we see that between two consecutive zeros $x_{k}$ and $x_{k+1} u(x)$ increases monotonically from $-\infty$ to $+\infty$. This allows us to define a set of functions $v_{k}$, the inverses of $u$, by

$$
v_{k}(u(x))=x \quad x_{k}<x<x_{k+1} .
$$

Note that $v_{k}(y)$ is defined for all $y$, it is smooth and monotonically increases from $x_{k}$ to $x_{k+1}$ as $y$ varies from $-\infty$ to $+\infty$. From (C.6) and the properties of the solution of Hill's equation it also follows that

$$
u\left(v_{k}(y)\right)=y \quad-\infty<y<\infty
$$

and

$$
v_{k+n}\left(e^{4 \pi b} y\right)=v_{k}(y)+2 \pi .
$$


We are now able to define the element $\alpha \in \widetilde{\operatorname{Diff}}_{0}\left(S^{1}\right)$ that transforms $\bar{\Psi}$ to $\Psi$ :

$$
\begin{array}{lc}
\alpha\left(x_{k}\right)=\bar{x}_{k+d} & k \in \mathbf{Z}, \\
\alpha(x)=\bar{v}_{k+d}(u(x)) & x_{k}<x<x_{k+1} .
\end{array}
$$

Here (and in the following) we use the convention that overlined objects are defined for $\bar{\Psi}$ analogously to the corresponding quantities for $\Psi$. Note the shift $k \rightarrow k+d$ in the index of the zeros. This shift has to be chosen so that the signs of $r_{k}$ and $\bar{r}_{k+d}$ coincide, which is clearly necessary for $\alpha$ to transform $\bar{\Psi}$ to $\Psi$.

Using (C.8) it is easy to show that $\alpha(x)$ as defined by (C.9) satisfies $\alpha(x+2 \pi)=$ $\alpha(x)+2 \pi$ and is smooth and strictly monotonically increasing for all $x \neq x_{k}$. To show that it is a proper conformal transformation, we still have to show that it is also differentiable at the zeros $x_{k}$.

Before we do that we differentiate (C.7) and use (C.4b) to obtain

$$
v_{k}^{\prime}(y)=\frac{1}{u^{\prime}\left(v_{k}(y)\right)}=\psi_{2}^{2}\left(v_{k}(y)\right)
$$

Using this (for the analogous overlined objects) and taking the derivative of the definition (C.9) we get

$$
\alpha^{\prime}(x)=\bar{\psi}_{2}^{2}\left(\bar{v}_{k+d}(u(x))\right) \frac{1}{\psi_{2}^{2}(x)}=\frac{\bar{\psi}_{2}^{2}(\alpha(x))}{\psi_{2}^{2}(x)} .
$$

Finally, on account of the equality of the sign of $r_{k}$ and $\bar{r}_{k+d}$, we can take the square root as

$$
\frac{1}{\sqrt{\alpha^{\prime}(x)}} \bar{\psi}_{2}(\alpha(x))=\psi_{2}(x) \quad x \neq x_{k},
$$

which is the required conformal transformation from $\bar{\Psi}$ to $\Psi$. More precisely, we still have to show that $\alpha$ is smooth and (C.12) holds also at the set of zeros $x_{k}$. We show this by first observing that (C.9) and (C.7) imply

$$
\bar{u}(\alpha(x))=\bar{u}\left(\bar{v}_{k+d}(u(x))\right)=u(x),
$$

which, when multiplied by (C.12), gives

$$
\frac{1}{\sqrt{\alpha^{\prime}(x)}} \bar{\psi}_{1}(\alpha(x))=\psi_{1}(x) \text {. }
$$

By continuity, (C.14) holds for all $x$ (including the set of zeros), and this allows us to express $\alpha^{\prime}$ as

$$
\alpha^{\prime}(x)=\frac{\bar{\psi}_{1}^{2}(\alpha(x))}{\psi_{1}^{2}(x)}
$$


showing that $\alpha(x)$ is indeed smooth also around the points $x_{k}$.

To summarize, we have proved that any solution vector of Hill's equation in the class $\mathcal{B}_{n}(b), n>0$ with monodromy matrix $B_{\eta}(b)$ can be expressed as the conformal transform of some unique representant, which can be chosen, for example, as in (3.58). Incidentally, the above consideration also clarifies the structure of the little group for the Virasoro orbit $\mathcal{B}_{n}(b)$. Because of the structure of $G\left[B_{\eta}(b)\right]$ in $(3.22 \mathrm{~b})$, we see that $\alpha \in \widetilde{\operatorname{Diff}_{0}}\left(S^{1}\right)$ belongs to the little group $\tilde{G}[\bar{L}]$ of $\bar{L}=\bar{\psi}_{2}^{\prime \prime} / \bar{\psi}_{2}$ if and only if it transforms $\bar{\psi}_{2}$ into itself up to a nonzero constant rescaling. The sign of this rescaling and the parity of the index shift $d$ in (C.9) are related and therefore the elements of $\tilde{G}[\bar{L}]$ can be labelled by the shift $d$ and the absolute value, $\lambda$, of the rescaling parameter. Since we can construct a unique element $\alpha_{\lambda, d} \in \tilde{G}[\bar{L}]$ for any $\lambda \in \mathbf{R}_{+}$and $d \in \mathbf{Z}$, the group $\tilde{G}[\bar{L}]$ is isomorphic to $\mathbf{R}_{+} \times \mathbf{Z}$, where $\mathbf{R}_{+}$is the multiplicative group of the positive real numbers. Factoring by the group of translations by multiples of $2 \pi$, whose elements are $\alpha_{\lambda, m n}$ with $\lambda=e^{-2 \pi b m}$ for $m \in \mathbf{Z}$, we obtain that $G[\bar{L}] \subset \operatorname{Diff}_{0}\left(S^{1}\right)$ is isomorphic to $\mathbf{R}_{+} \times \mathbf{Z}_{n}$.

Our considerations are also valid in the parabolic case $\mathcal{P}_{n}^{q}$ if we replace (C.4a) by

$$
u(x+2 \pi)=u(x)+q
$$

and (C.8) by

$$
v_{k+n}(y+q)=v_{k}(y)+2 \pi .
$$

For any $L$ on the Virasoro orbit $\mathcal{P}_{n}^{q}$ with $n>0$, the little groups $\tilde{G}[L]$ and $G[L]$ are again isomorphic to $\mathbf{R}_{+} \times \mathbf{Z}$ and to $\mathbf{R}_{+} \times \mathbf{Z}_{n}$, respectively. 


\section{Appendix D: The connectedness of the phase space with $2 n$ zeros}

In this Appendix we show that beyond the number of zeros, which is a topological invariant as discussed in Appendix A, there are no additional topological invariants in the solution space of the global Liouville equation. In other words, any two solutions of the global Liouville equation with the same number of zeros can be continuously connected along a path of solutions. (In the case of no zeros the two solutions must also be of the same sign).

To show this we first note that any solution can be continuously deformed into one of the representatives listed in Section 5. Indeed, any solution is a conformal transform of one of the representatives and the conformal group as defined in (1.10) is connected. For the case of no zeros the representatives are given by (5.11) and it is clear that apart from the overall sign they are all connected (by changing $b$ along the first vertical line of Figure 1).

In the case of $N=2 n \neq 0$ zeros there are representatives with elliptic, $E$-type, hyperbolic and parabolic monodromy. It is clear that the elliptic representatives (given by (5.4)) together with the $E$-type representatives of (5.7) are connected (by changing $\nu_{+}=N-\nu_{-}$along the horizontal line of Figure 1). On the other hand, the hyperbolic and parabolic representatives with $N=n_{+}+n_{-}$where neither $n_{+}$nor $n_{-}$vanishes are easily connected to the corresponding $E$-type representatives. For the hyperbolic case the connection is along the vertical lines of Figure 1, since the representatives (5.16) are simply reduced to $(5.7)$ in the limit $b \rightarrow 0$. For the parabolic case of $(5.20)$ we have to move along the curve (3.77) parametrized by $a$ from $a=1$ to $a=\infty$, i.e. from (5.20) to $(5.7)$.

Hyperbolic and parabolic solutions with $n_{+}=0$ (and the analogous $n_{-}=0$ cases) are more difficult to connect with the elliptic ones and here we need a different way of connecting the solutions. (The reason why a simple connection along Figure 1 is not available for these cases is the existence of the empty circle at $n=0$ which blocks the way between the first vertical line and the horizontal line of the figure.)

We will make use of a different way of representing the solutions of the global Liouville equation, namely, by considering it to be an initial-value problem. It is clear that a solution can be uniquely represented by its value and first time derivative at $\tau=0$ :

$$
q(\sigma)=Q(0, \sigma) \quad \text { and } \quad k(\sigma)=\frac{\partial}{\partial \tau} Q(0, \sigma)
$$

It would be possible to connect any two solutions, $\left\{q_{1}, k_{1}\right\}$ and $\left\{q_{2}, k_{2}\right\}$ along a straight line:

$$
\begin{aligned}
& q(\sigma, t)=(1-t) q_{1}(\sigma)+t q_{2}(\sigma) \\
& k(\sigma, t)=(1-t) k_{1}(\sigma)+t k_{2}(\sigma)
\end{aligned}
$$


for $t$ between 0 and 1 if it did not follow from the global Liouville equation (1.1) that at all points where $q(\sigma)$ vanishes,

$$
\left(q^{\prime}\right)^{2}=1+k^{2}
$$

must hold. However, it is clear that the pair of functions (D.2) does not satisfy (D.3) in general (only for $t=0,1$ ) and therefore (D.2) does not represent a curve in the space of solutions.

If, on the other hand, the two solutions are such that the set of zeros, $\left\{x_{1}, \ldots, x_{2 n}\right\}$ is the same for $q_{1}$ and $q_{2}$ and at each zero

$$
q_{1}^{\prime}=q_{2}^{\prime} \quad \text { and } \quad k_{1}=k_{2},
$$

hold, then (D.2) can after all be used to connect the two solutions in the space of solutions.

Let us consider now the solutions (5.15). The corresponding pair, $\{q, k\}$, has the following properties: $q(\sigma)$ has $2 n$ zeros at equal $(2 \pi / n)$ spacing. At each of the zeros we have

$$
\begin{aligned}
\operatorname{sign}\left(q^{\prime} k\right) & =-1, \\
\left|q^{\prime}\right| & =\frac{f+1}{\sqrt{2 f+1}}, \\
|k| & =\frac{f}{\sqrt{2 f+1}},
\end{aligned}
$$

where

$$
f=\frac{n-\frac{3 b}{2}+\frac{b^{2}}{8 n}}{2 b} .
$$

The $\{q, k\}$ pair corresponding to the parabolic solution (5.19) has similar properties, namely, $q$ has also $2 n$ equally spaced zeros and (D.5) holds at the zeros with

$$
f=\pi-1 .
$$

Finally, for the elliptic case, using (5.4), we find that at the similarly equally spaced zeros (D.5) holds with

$$
f=\frac{n}{\nu_{+}}-1 .
$$

Thus, the parabolic and the hyperbolic solutions can be connected with the elliptic ones using (D.2) if for the latter we choose

$$
\nu_{+}=\frac{n}{\pi} \quad \text { and } \quad \nu_{+}=\frac{2 b n}{n+\frac{b}{2}+\frac{b^{2}}{8 n}}
$$

respectively. 


\section{References}

[1] N. Seiberg, Prog. Theor. Phys. Suppl. 102 (1990) 319.

[2] G.P. Dzhordzhadze, A.K. Pogrebkov and M.C. Polivanov, Theor. Math. Phys. 40(2) (1980) 706; A.K. Pogrebkov and M.K. Polivanov, Sov. Sci. Rev. C (Math. Phys.) Vol. 5 (1985) pp. 197-272, and references therein.

[3] L.Johansson, A. Kihlberg and R. Marnelius, Phys. Rev. D29 (1984) 2798; L. Johannson and R. Marnelius, Nucl. Phys. B254 (1985) 201.

[4] J. Balog, L. Fehér, P. Forgács, L. O'Raifeartaigh and A. Wipf, Phys. Lett. B227 (1989) 214; Ann. Phys. (N.Y.) 203 (1990) 76.

[5] E. Witten, Commun. Math. Phys. 92 (1984) 483.

[6] L. Fehér and I. Tsutsui, Prog. Theor. Phys. Suppl. 118 (1995) 173; J. Geom. Phys. 21 (1997) 97.

[7] W. Magnus and S. Winkler, Hill's Equation, Interscience, 1966 (Dover, 1979).

[8] V.P. Lazutkin and T.F. Pankratova, Funct. Anal. Appl. 9(4) (1975) 41.

[9] A.A. Kirillov, Funct. Anal. Appl. 15(2) (1981) 135; Springer Lecture Notes in Mathematics, vol. 970 (1982) 101.

[10] G. Segal, Commun. Math. Phys. 80 (1981) 301.

[11] E. Witten, Commun. Math. Phys. 114 (1988) 1.

[12] V.Yu. Ovsienko and B.A. Khesin, Funct. Anal. Appl. 24(1) (1990) 33.

[13] A. Gorsky, B. Roy and K. Selivanov, JETP Lett. 53(1) (1991) 64.

[14] L. Guieu, Sur la geometrie des orbites de la representation coadjointe du groupe de Bott-Virasoro, PhD thesis, Universite d'Aix-Marseille I, 1994.

[15] L.D. Faddeev and L. Takhtajan Springer Lecture Notes in Physics, vol. 246 (1986) 166.

[16] E. Aldrovandi, L. Bonora, V. Bonservizi and R. Paunov, Int. J. Mod. Phys. A9 (1994) 57.

[17] J. Liouville, J. Math. Pures Appl. 18 (1853) 71.

[18] E. Braaten, T. Curtright and C. Thorn, Phys. Lett. B118 (1982) 115; Ann. Phys. (N.Y.) 147 (1983) 365; T. Curtright and C. Thorn, Phys. Rev. Lett. 48 (1982) 1309.

[19] H.J. Otto and G. Weigt Phys. Lett. B159 (1985) 341; Z. Phys. C31 (1986) 219.

[20] J.L. Gervais and A. Neveu, Nucl. Phys. B199 (1982) 59; ibid B209 (1982) 125; ibid B224 (1983) 329; J.L. Gervais and J. Schnittger, Nucl. Phys. B413 (1994) 433. 
[21] A. Gorsky and A. Johansen, Int. J. Mod. Phys. A10 (1995) 785.

[22] T. Fülöp, J. Math. Phys. 37 (1996) 1617.

[23] H. Kobayashi and I. Tsutsui, Nucl. Phys. B472 (1996) 409.

[24] A.V. Razumov and V.I. Yasnov, Hamiltonian reduction of free particle motion on group $S L(2, \mathbf{R})$, preprint hep-th/9609030.

[25] Z. Bajnok, L. Palla and G. Takács, Nucl. Phys. B385 (1992) 329.

[26] B.A. Khesin and B.Z. Shapiro, Commun. Math. Phys. 145 (1992) 357. 